\title{
Some New Logarithmic Aggregation Operators and their Application to Group Decision Making Problem Based on t-Norm and t-Conorm
}

khaista Rahman ( $\sim$ khaista355@yahoo.com )

Shaheed Benazir Bhutto University Sheringal

\section{Research Article}

Keywords: logarithmic operational laws, Averaging operators, geometric operators.

Posted Date: November 22nd, 2021

DOI: https://doi.org/10.21203/rs.3.rs-1072220/v1

License: (c) (i) This work is licensed under a Creative Commons Attribution 4.0 International License.

Read Full License

Version of Record: A version of this preprint was published at Soft Computing on February 7th, 2022. See the published version at https://doi.org/10.1007/s00500-022-06730-8. 


\title{
Some New Logarithmic Aggregation Operators and their Application to Group Decision Making Problem Based on t-Norm and t-Conorm
}

\author{
Khaista Rahman \\ Department of Mathematics Shaheed Benazir Bhutto University Sheringal, Pakistan \\ khaista355@yahoo.com
}

\begin{abstract}
In this paper, a logarithmic operational law for intuitionistic fuzzy numbers is defined, in which the based $l$ is a real number such that $l \in(0,1)$ with condition $l \neq 1$. Some properties of logarithmic operational laws have been studied and based on these, several Einstein averaging and Einstein geometric operators namely, logarithmic intuitionistic fuzzy Einstein weighted averaging (LIFEWA) operator, logarithmic intuitionistic fuzzy Einstein ordered weighted averaging (LIFEOWA) operator, logarithmic intuitionistic fuzzy Einstein hybrid averaging (LIFEHA) operator, logarithmic intuitionistic fuzzy Einstein weighted geometric (LIFEWG) operator, logarithmic intuitionistic fuzzy Einstein ordered weighted geometric (LIFEOWG) operator, and logarithmic intuitionistic fuzzy Einstein hybrid geometric (LIFEHG) operator have been introduced, which can overcome the weaknesses of algebraic operators. Furthermore, based on the proposed operators a multi-attribute group decision-making problem is established under logarithmic operational laws. Finally, an illustrative example is used to illustrate the applicability and validity of the proposed approach and compare the results with the existing methods to show the effectiveness of it.
\end{abstract}

Keywords: logarithmic operational laws, Averaging operators, geometric operators.

\section{Introduction}

Multi-attribute group decision-making (MCDM) is one of the successful developing study active problems recently for a last decision within a time. However it is not always acceptable to give the favorites in a specific method due to many restrictions and hence their analogous results are not best in some environments. To solve this challenging type problem, Zadeh (1965) presented the idea of fuzzy set (FS), which has only one element called membership function. Zadeh was the first to studied and develop the solution of different difficult type problems with the help of FS. After their prosperous and successful application Atanassov (1986, 1989), generalized this concept and improve the notion of FS to IFS. IFS having element in the ordered pair form. IFS are the efficacious and valuable generalization of 
FS. IFS is the more suitable and powerful method for the solution of problems. Under this situation, there are several researchers functioned in IFSs area and presented some different methods and operators for the solution of the problems. Yager and Kacprzyk (1997) presented some basic and an important and role for fusion procedure. Yager (1988) presented OWA operator. Xu and Yager (2006) explored the notion of IFWG operator, IFOWG operator and IFHG operator. Xu (2007) explored the idea of weighed averaging operator, such as the IFWA operator, the IFOWA operator and the IFHA operator based on IFNs. Wang and Liu (2011, 2012) explored the notion of several operators, such as the IFEWG operator, the IFEOWG operator, the IFEWA operator, the IFEOWA operator based on Einstein operations and applied these operators on decision making problems. Ye $(2007,2009)$ using IFS and developed the notion of a new accuracy degree for interval-valued and developed MAGDM based on vague sets. Zhao and Wei (2013) familiarized the concept of some Einstein hybrid operators namely, the IFEHA operator, the IFEHG operator and their weight and advantages in real life problems. Xu et.al (2014) explored the notion of Einstein Choquet integral based on IFNs. Garg (2016a, 2016b, 2016c, 2016d) explored the idea of score function, accuracy degree for IVIFVs for ranking methods, and also developed many operators for decision making problems. After the producing of these ideas, Garg and Nancy (2016d), Nancy and Garg (2016e), presented some more operators for MAGDM problems. Dalman et al. (2016), Yu (2014, 2015), Yu and Shi (2015), Kumar and Garg (2016), Garg et al. (2015) introduced several operators and their basic application in decision making in real life problems. Rahman et al. (2018) established the notion of generalized operators. Gou (2016) established the idea of exponential operational laws and develop several operators using IFS and IFNs. Meng et al. (2019), Yang et al. (2021), Li et al. (2021), Ding et al. (2020), Kordestani et al. (2021), He et al. (2020), Zhang et al. (2021), Zhao et al. (2020), Sun et al. (2021), Sun et al. (2015) introduced many new methods related to fuzzy set theory and intuitionistic fuzzy set theory. After the positive applications of exponential operational laws (EOL) it was necessary to be developing the idea of logarithmic operational laws (LOL). Li and Wei (2017) introduced the notion of logarithmic operational law and develop some new logarithmic operators, such as the LIFWA operator, the LIFOWA operator, the LIFWG operator, the LIFOWG operator. For developing the stated methods some numerical examples are constructed. They also developed relationship between exponential operational law and logarithmic operational law. 
1.1) Motivation: Motivated from the methods ( $\mathrm{Li}$ and $\mathrm{Wei}, 2017$ ), in which the authors introduced some algebraic operations, and then developed some algebraic operators based on these operations. However, we know that Einstein sum and Einstein product are the good alternative of algebraic sum and product. Therefore in this paper, we introduced some novel Einstein operations, which can overcome the weaknesses of algebraic operations, and then developed several Einstein aggregation operators, which can overcome the algebraic operator's drawbacks.

1.2) Contribution: The main contributions of our paper as follows:

i) We introduced some new logarithmic operations for intuitionistic fuzzy sets, which can overcome the weaknesses of algebraic operations and capture the relationship between various intuitionistic fuzzy sets.

ii) We developed some logarithmic operators named as LIFEWA operator, LIFEOWA operator, LIFEHA operator, LIFEWG operator, LIFEOWG operator, and LIFEHG operator for IFSs, which can overcome the algebraic operator's drawbacks.

iii) We developed an algorithm to deal with multi-attribute decision making problems under intuitionistic fuzzy information.

iv) To show the effectiveness and reliability of the proposed logarithmic aggregation operators, we applied the proposed operator to a MAGDM problem.

v) The results indicate that the proposed technique is more effective and provides a more accurate output compared to existing methods.

1.3) Organization: The rest of the paper is organized as. In section 2, we develop some basic definitions. In section 3, we introduce some operational laws. In section 4, we introduce some operators namely, LIFEWA operator, LIFEOWA operator, LIFEHA operator, LIFEWG operator, LIFEOWG operator and LIFEHG operator. In section 5, we apply the propose operators to decision making. In section 6, we develop an numerical example to select the best option. In section 7 , we have limitations. In section 8 , we have conclusion.

\section{Preliminaries}

In this section, we present some basic definitions related with IFSs and some algebraic operators named as LIFWA operator, LIFOWA operator, LIFWG operator, LIFOWG operator. 
Definition 1 (Lia and Wei, 2017) Let $Z$ be a fixed set and $\mathfrak{R}=\left\{\left\langle z, r_{\mathfrak{R}}(z), h_{\mathfrak{R}}(z)\right\rangle \mid z \in Z\right\}$ be an IFS, and then logarithmic operational law can be defined as follows:

$$
\log _{1} \Re=\left\{\left\langle z, 1-\log _{1} r_{\mathfrak{R}}(z), \log _{1}\left(1-h_{\Re}(z)\right)\right\rangle \mid z \in Z\right\}, 0 \text { p } 1 \leq r \leq 1,1 \neq 1
$$

where $1-\log _{1} r_{\mathfrak{R}}(z): Z \rightarrow[0,1], \forall z \in Z \rightarrow 1-\log _{1} r_{\mathfrak{R}}(z) \in[0,1]$ is called the membership degree (MD) and $1-\log _{1} h_{\mathfrak{R}}(z): Z \rightarrow[0,1], \forall z \in Z \rightarrow 1-\log _{1} h_{\mathfrak{R}}(z) \in[0,1]$ is called the nonmembership degree (NMD) with condition $0 \leq 1-\log _{1} r_{\mathfrak{R}}(z)+\log _{1}\left(1-h_{\mathfrak{R}}(z)\right) \leq 1$. Let $1-\left(1-\log _{1} r_{\mathfrak{R}}(z)+\log _{1}\left(1-h_{\mathfrak{R}}(z)\right)\right)$, then it is called the degree of indeterminacy.

Definition 2 (Xu and Yager, 2006): Let $\rho=\left(r_{\rho}, h_{\rho}\right)$ be an IFN, and $\log _{1} \mathfrak{R}=\left\{\left\langle z, 1-\log _{1} r_{\mathfrak{R}}(z), \log _{1}\left(1-h_{\mathfrak{R}}(z)\right)\right\rangle \mid z \in Z\right\}$, then $\log _{1} \Re$ is called logarithmic operator and the value of $\log _{1} \mathfrak{R}$ is called logarithmic intuitionistic fuzzy number.

Definition 3 (Xu and Yager, 2006): Let $\rho=\left(r_{\rho}, h_{\rho}\right)$ be an IFV, then the score function and accuracy degree can be stated as: $S(\rho)=r_{\rho}-h_{\rho}$ with condition $S(\rho) \in[-1,1]$ and $H(\rho)=r_{\rho}+h_{\rho}$ with condition $H(\rho) \in[0,1]$ respectively.

Definition 4 (Xu and Yager, 2006): Let $\rho_{1}=\left(r_{\rho_{1}}, h_{\rho_{1}}\right), \rho_{2}=\left(r_{\rho_{2}}, h_{\rho_{2}}\right)$ are two IFVs, then

i) If $S\left(\rho_{2}\right) \mathrm{f} S\left(\rho_{1}\right)$, then $\rho_{1} \mathrm{p} \rho_{2}$

ii) If $S\left(\rho_{1}\right)=S\left(\rho_{2}\right)$, then we have two cases:

a) If $H\left(\rho_{1}\right)=H\left(\rho_{2}\right)$, then $\rho_{1}=\rho_{2}$

b) If $H\left(\rho_{1}\right) \mathrm{p} H\left(\rho_{2}\right)$, then $\rho_{1} \mathrm{p} \rho_{2}$

Definition 5 (Xu and Yager, 2006): Let $\rho=\left(r_{\rho}, h_{\rho}\right), \rho_{1}=\left(r_{\rho_{1}}, h_{\rho_{1}}\right), \rho_{2}=\left(r_{\rho_{2}}, h_{\rho_{2}}\right)$ are any three IFVs, and $t \mathrm{f} 0$, then the following conditions hold:

i) $\quad \rho^{c}=\left(h_{\rho}, r_{\rho}\right)$

ii) $\quad \rho_{1} \leq \rho_{2}$ if and only if $r_{1} \leq r_{2}$ and $h_{1} \geq h_{2}$

iii) $\quad \rho_{1}=\rho_{2}$ if and only if $\rho_{1} \leq \rho_{2}$ and $\rho_{2} \leq \rho_{1}$ 
iv) $\quad \rho_{1} \cup \rho_{2}=\left(\max \left\{r_{\rho_{1}}, r_{\rho_{2}}\right\}, \min \left\{h_{\rho_{1}}, h_{\rho_{2}}\right\}\right)$

v) $\quad \rho_{1} \cap \rho_{2}=\left(\min \left\{r_{\rho_{1}}, r_{\rho_{2}}\right\}, \max \left\{h_{\rho_{1}}, h_{\rho_{2}}\right\}\right)$

vi) $\quad \rho_{1} \oplus_{\varepsilon} \rho_{2}=\left(\frac{r_{\rho_{1}}+r_{\rho_{2}}}{1+r_{\rho_{1}} r_{\rho_{2}}}, \frac{h_{\rho_{1}} h_{\rho_{2}}}{1+\left(1-h_{\rho_{1}}\right)\left(1-h_{\rho_{2}}\right)}\right)$

vii) $\quad \rho_{1} \otimes_{\varepsilon} \rho_{2}=\left(\frac{r_{\rho_{1}} r_{\rho_{2}}}{1+\left(1-r_{\rho_{1}}\right)\left(1-r_{\rho_{2}}\right)}, \frac{h_{\rho_{1}}+h_{\rho_{2}}}{1+h_{\rho_{1}} h_{\rho_{2}}}\right)$

viii) $\quad(\rho)^{t}=\left(\frac{2\left(r_{\rho}\right)^{t}}{\left(2-r_{\rho}\right)^{t}+\left(r_{\rho}\right)^{t}}, \frac{\left(1+h_{\rho}\right)^{t}-\left(1-h_{\rho}\right)^{t}}{\left(1+h_{\rho}\right)^{t}+\left(1-h_{\rho}\right)^{t}}\right)$

ix) $\quad t(\rho)=\left(\frac{\left(1+r_{\rho}\right)^{t}-\left(1-r_{\rho_{1}}\right)^{t}}{\left(1+r_{\rho}\right)^{t}+\left(1-r_{\rho}\right)^{t}}, \frac{2\left(h_{\rho}\right)^{t}}{\left(2-h_{\rho}\right)^{t}+\left(h_{\rho}\right)^{t}}\right)$

х) $\quad(1)^{\rho}= \begin{cases}(1)^{1-r}, \quad 1-1^{h} & 1 \in(0,1) \\ \left(\frac{1}{1}\right)^{1-r}, 1-\left(\frac{1}{1}\right)^{h} & 1 \geq 1\end{cases}$

Definition 6: (Lia and Wei, 2017): The LIFWA operator can be stated as:

$$
\operatorname{LIFWA}_{s}\left(\rho_{1}, \rho_{2}, \ldots, \rho_{n}\right)=\left(1-\prod_{j=1}^{n}\left(\log _{1_{j}} r_{j}\right)^{s_{j}}, \prod_{j=1}^{n}\left(\log _{1_{j}}\left(1-h_{j}\right)\right)^{s_{j}}\right)
$$

where $s=\left(s_{1}, s_{2}, \ldots, s_{n}\right)^{T}$ be the weighted vector with condition $s_{j} \in[0,1]$ and $\sum_{j=1}^{n} s_{j}=1$.

Definition 7: (Lia and Wei, 2017): The LIFOWA operator can be defined as:

$$
\operatorname{LIFOWA}_{s}\left(\rho_{1}, \rho_{2}, \ldots \rho_{n}\right)=\left(1-\prod_{j=1}^{n}\left(\log _{1_{\tau(j)}} r_{\tau(j)}\right)^{s_{j}}, \prod_{j=1}^{n}\left(\log _{\tau(j)}\left(1-h_{\tau(j)}\right)\right)^{s_{j}}\right)
$$

where $s=\left(s_{1}, s_{2}, \ldots, s_{n}\right)^{T}$ be the weighted vector with condition $s_{j} \in[0,1], \sum_{j=1}^{n} s_{j}=1, \quad$ and $(\tau(1), \tau(2), \ldots, \tau(n))$ is any rearrangement of $(1,2, \ldots, n)$. 
Definition 8: (Lia and Wei, 2017): The LIFWG operator can be defined as:

$$
\operatorname{LIFWG}_{s}\left(\rho_{1}, \rho_{2}, \ldots, \rho_{n}\right)=\left(\prod_{j=1}^{n}\left(1-\log _{1_{j}} r_{j}\right)^{s_{j}}, 1-\prod_{j=1}^{n}\left(1-\log _{1_{j}}\left(1-h_{j}\right)\right)^{s_{j}}\right)
$$

where $s=\left(s_{1}, s_{2}, \ldots, s_{n}\right)^{T}$ be the weighted vector with condition $s_{j} \in[0,1]$ and $\sum_{j=1}^{n} s_{j}=1$.

Definition 9: (Lia and Wei, 2017): The LIFOWG operator can be defined as:

$$
\operatorname{LIFOWG}_{s}\left(\rho_{1}, \rho_{2}, \ldots, \rho_{n}\right)=\left(\prod_{j=1}^{n}\left(1-\log _{\tau(j)} r_{\tau(j)}\right)^{s_{j}}, 1-\prod_{j=1}^{n}\left(1-\log _{\tau(j)}\left(1-h_{\tau(j)}\right)\right)^{s_{j}}\right)
$$

where $s=\left(s_{1}, s_{2}, \ldots, s_{n}\right)^{T}$ be the weighted vector with condition $s_{j} \in[0,1], \sum_{j=1}^{n} s_{j}=1, \quad$ and $(\tau(1), \tau(2), \ldots, \tau(n))$ is any rearrangement of $(1,2, \ldots, n)$.

\section{Some new Logarithmic Operational laws for IFNs}

In this part, we introduce the notion of several logarithmic operational laws with IFNs.

Theorem 1: Let $\rho=(r, h)$ be an IFN, if 0 p $1 \leq \min \{r,(1-h)\} \leq 1,1 \neq 1$. Then $1^{\log _{1} \rho}=\rho$.

Proof: Since utilize the above given information, then we get:

$$
\begin{aligned}
1^{\log _{1} \rho} & =\left(1-\left(1-\log _{1}\left\{\frac{(1+r)-(1-r)}{(1+r)+(1-r)}\right\}\right), 1-1 \log _{1}\left\{\frac{1-h}{(2+h)-(1+h)}\right\}\right) \\
& =\left(1^{1-1+\log _{1}\left\{\frac{(1+r)-(1-r)}{(1+r)+(1-r)}\right\}, 1-1} \log _{1}\left\{\frac{1-h}{(2+h)-(1+h)}\right\}\right) \\
& =\left(\frac{(1+r)-(1-r)}{(1+r)+(1-r)}, 1-\frac{1-h}{(2+h)-(1+h)}\right)=(r, h)=\rho
\end{aligned}
$$

Theorem 2: Let $\rho=(r, h)$ be an IFN, if 0 p $1 \leq \min \{r,(1-h)\} \leq 1,1 \neq 1$. Then $\log _{1} 1^{\rho}=\rho$ Proof: Since utilize the above given information, then we get: 


$$
\begin{aligned}
\log _{1} 1^{\rho} & \left.=\log _{1}\left(1-\left(\frac{(1+r)-(1-r)}{(1+r)+(1-r)}\right), 1-1\right)^{\frac{1-h}{(2+h)-(1+h)}}\right) \\
& =\left(1-\log _{1} 1^{1-\left(\frac{(1+r)-(1-r)}{(1+r)+(1-r)}\right)}, \log _{1}\left(1-\left(1-1^{\frac{1-h}{(2+h)-(1+h)}}\right)\right)\right) \\
& =\left(1-1+\left(\frac{(1+r)-(1-r)}{(1+r)+(1-r)}\right), \frac{1-h}{(2+h)-(1+h)}\right)=(r, h)=\rho
\end{aligned}
$$

Theorem 3: Let $\rho_{j}=\left(r_{j}, h_{j}\right)$ be a family of three IFNs with $0 \mathrm{p} 1 \leq \min \{r,(1-h)\} \leq 1,1 \neq 1$ and $1 \neq 1$. Then the following properties hold:

1) $\log _{1} \rho_{1} \oplus_{\varepsilon} \log _{1} \rho_{2}=\log _{1} \rho_{2} \oplus_{\varepsilon} \log _{1} \rho_{1}$

2) $\log _{1} \rho_{1} \otimes_{\varepsilon} \log _{1} \rho_{2}=\log _{1} \rho_{2} \otimes_{\varepsilon} \log _{1} \rho_{1}$

3) $\left(\log _{1} \rho_{1} \oplus_{\varepsilon} \log _{1} \rho_{2}\right) \oplus_{\varepsilon} \log _{1} \rho_{3}=\log _{1} \rho_{1} \oplus_{\varepsilon}\left(\log _{1} \rho_{2} \oplus_{\varepsilon} \log _{1} \rho_{3}\right)$

4) $\left(\log _{1} \rho_{1} \otimes_{\varepsilon} \log _{1} \rho_{2}\right) \otimes_{\varepsilon} \log _{1} \rho_{3}=\log _{1} \rho_{1} \otimes_{\varepsilon}\left(\log _{1} \rho_{2} \otimes_{\varepsilon} \log _{1} \rho_{3}\right)$

Proof: As $\rho_{1}, \rho_{2}, \rho_{3}$ are three IFNs, then we have

$$
\log _{1} \rho_{1}=\left(1-\log _{1} r_{1}, \log _{1}\left(1-h_{1}\right)\right)
$$

and

$$
\log _{1} \rho_{2}=\left(1-\log _{1} r_{2}, \log _{1}\left(1-h_{2}\right)\right)
$$

1) Since

$$
\begin{aligned}
& \log _{1} \rho_{1} \oplus_{\varepsilon} \log _{1} \rho_{2} \\
& =\left(1-\log _{1} r_{1}, \log _{1}\left(1-h_{1}\right)\right) \oplus_{\varepsilon}\left(1-\log _{1} r_{2}, \log _{1}\left(1-h_{2}\right)\right) \\
& =\left(\frac{1-\log _{1} r_{1}+1-\log _{1} r_{2}}{1+\left(1-\log _{1} r_{1}\right)\left(1-\log _{1} r_{2}\right)}, \frac{\log _{1}\left(1-h_{1}\right) \log _{1}\left(1-h_{2}\right)}{1+\left(1-\log _{1}\left(1-h_{1}\right)\right)\left(1-\log _{1}\left(1-h_{2}\right)\right)}\right) \\
& =\left(\frac{1-\log _{1} r_{2}+1-\log _{1} r_{1}}{1+\left(1-\log _{1} r_{2}\right)\left(1-\log _{1} r_{1}\right)}, \frac{\log _{1}\left(1-h_{2}\right) \log _{1}\left(1-h_{1}\right)}{1+\left(1-\log _{1}\left(1-h_{2}\right)\right)\left(1-\log _{1}\left(1-h_{1}\right)\right)}\right) \\
& =\log _{1} \rho_{2} \oplus_{\varepsilon} \log _{1} \rho_{1}
\end{aligned}
$$

2) Since 


$$
\begin{aligned}
& \log _{1} \rho_{1} \otimes_{\varepsilon} \log _{1} \rho_{2} \\
& =\left(1-\log _{1} r_{1}, \log _{1}\left(1-h_{1}\right)\right) \otimes_{\varepsilon}\left(1-\log _{1} r_{2}, \log _{1}\left(1-h_{2}\right)\right) \\
& =\left(\frac{\left(1-\log _{1} r_{1}\right)\left(1-\log _{1} r_{2}\right)}{1+\left(1-\left(1-\log _{1} r_{1}\right)\right)\left(1-\left(1-\log _{1} r_{2}\right)\right)}, \frac{\log _{1}\left(1-h_{1}\right) \oplus \log _{1}\left(1-h_{2}\right)}{1+\log _{1}\left(1-h_{1}\right) \log _{1}\left(1-h_{2}\right)}\right) \\
& =\left(\frac{\left(1-\log _{1} r_{2}\right)\left(1-\log _{1} r_{1}\right)}{1+\left(1-\left(1-\log _{1} r_{2}\right)\right)\left(1-\left(1-\log _{1} r_{1}\right)\right)}, \frac{\log _{1}\left(1-h_{2}\right) \oplus \log _{1}\left(1-h_{1}\right)}{1+\log _{1}\left(1-h_{2}\right) \log _{1}\left(1-h_{1}\right)}\right) \\
& =\log _{1} \rho_{2} \otimes_{\varepsilon} \log _{1} \rho_{1}
\end{aligned}
$$

The proof of (3) and (4) are similar to (1), (2) respectively, so here these proofs are omitted.

Theorem 4: Let $\rho_{j}=\left(r_{j}, h_{j}\right)(j=1,2)$ be a collection of any two IFNs such as, $0 \mathrm{p}{ }_{j} \leq \min \left\{r_{j},\left(1-h_{j}\right)\right\} \leq 1,1_{j} \neq 1$, and $\kappa \mathrm{f} 0$. Then the following properties hold:
1) $\kappa\left(\log _{1} \rho_{1} \oplus_{\varepsilon} \log _{1} \rho_{2}\right)=\kappa \log _{1} \rho_{1} \oplus_{\varepsilon} \kappa \log _{1} \rho_{2}$
2) $\left(\log _{1} \rho_{1} \otimes_{\varepsilon} \log _{1} \rho_{2}\right)^{K}=\left(\log _{1} \rho_{1}\right)^{K} \otimes_{\varepsilon}\left(\log _{1} \rho_{2}\right)^{K}$
3) $\kappa_{1} \log _{1} \rho \oplus_{\varepsilon} \kappa_{2} \log _{1} \rho=\left(\kappa_{1}+\kappa_{2}\right) \log _{1} \rho$
4) $\left(\log _{1} \rho\right)^{K_{1}} \otimes_{\mathcal{E}}\left(\log _{1} \rho\right)^{K_{2}}=\left(\log _{1} \rho\right)^{\kappa_{1}+\kappa_{2}}$
5) $\left(\left(\log _{1} \rho\right)^{\kappa_{1}}\right)^{\kappa_{2}}=\left(\log _{1} \rho\right)^{\kappa_{1} \kappa_{2}}$

Proof: the proof is easy, so it is omitted here.

\section{Some Logarithmic Einstein Aggregation operators}

In this section, we develop several logarithmic Einstein aggregation operators based on IFNs namely, LIFEWA operator, LIFEOWA operator, LIFEHA operator, LIFEWG operator, LIFEOWG operator, and LIFEHG operator along with their properties such as, idempotency, boundedness, monotonicity.

Definition 10: Let $\rho_{j}=\left(r_{j}, h_{j}\right)(j=1,2, \ldots, n)$ be a group of $n$ IFVs, and $s=\left(s_{1}, s_{2}, s_{3}, \ldots, s_{n}\right)^{T}$ be their weighted vector with some condition such as, $s_{j} \in[0,1]$ and $\sum_{j=1}^{n} s_{j}=1$. Then the LIFEWA operator can be defined as: 


$$
\begin{aligned}
& \operatorname{LIFEWA}_{s}\left(\rho_{1}, \rho_{2}, \ldots, \rho_{n}\right) \\
& \int\left(\frac{\prod_{j=1}^{n}\left(2-\log _{1_{j}} r_{j}\right)^{s_{j}}-\prod_{j=1}^{n}\left(\log _{1_{j}} r_{j}\right)^{s_{j}}}{\prod_{j=1}^{n}\left(2-\log _{1_{j}} r_{j}\right)^{s_{j}}+\prod_{j=1}^{n}\left(\log _{1_{j}} r_{j}\right)^{s_{j}}}, \frac{2 \prod_{j=1}^{n}\left(\log _{1_{j}}\left(1-h_{j}\right)\right)^{s_{j}}}{\prod_{j=1}^{n}\left(2-\log _{1_{j}}\left(1-h_{j}\right)\right)^{s_{j}}+\prod_{j=1}^{n}\left(\log _{1_{j}}\left(1-h_{j}\right)\right)^{s_{j}}}\right) \\
& \text { where } 0 \mathrm{p} 1_{j} \leq \min \left\{r_{j},\left(1-h_{j}\right)\right\} \leq 1 ; 1_{j} \neq 1
\end{aligned}
$$

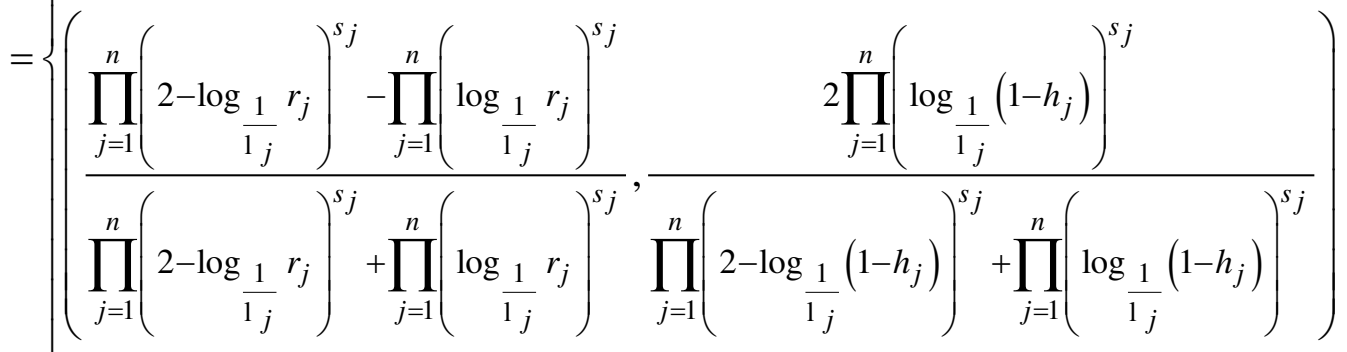

$$
\begin{aligned}
& \text { where } 0 \mathrm{p} \frac{1}{1_{j}} \leq \min \left\{r_{j},\left(1-h_{j}\right)\right\} \leq 1 ; 1_{j} \neq 1
\end{aligned}
$$

Theorem 5: Let $\rho_{j}=\left(r_{j}, h_{j}\right)$ be a group of $n$ IFVs , then their resulting value by using LIFEWA operator is also a IFV, and

$$
\begin{aligned}
& \operatorname{LIFEWA}_{s}\left(\rho_{1}, \rho_{2}, \rho_{3}, \ldots, \rho_{n}\right) \\
& =\left(\frac{\prod_{j=1}^{n}\left(2-\log _{1_{j}} r_{j}\right)^{s_{j}}-\prod_{j=1}^{n}\left(\log _{1_{j}} r_{j}\right)^{s j}}{\prod_{j=1}^{n}\left(2-\log _{1_{j}} r_{j}\right)^{s_{j}}+\prod_{j=1}^{n}\left(\log _{1_{j}} r_{j}\right)^{s j}}, \frac{2 \prod_{j=1}^{n}\left(\log _{1_{j}}\left(1-h_{j}\right)\right)^{s_{j}}}{\prod_{j=1}^{n}\left(2-\log _{1_{j}}\left(1-h_{j}\right)\right)^{s_{j}}+\prod_{j=1}^{n}\left(\log _{1_{j}}\left(1-h_{j}\right)\right)^{s_{j}}}\right)
\end{aligned}
$$

Proof: The given result can be showed by mathematical induction. For $n=2$.

$$
s_{1} \rho_{1}=\left(\frac{\left(2+\log _{1_{1}}\left(r_{1}\right)\right)^{s_{1}}-\left(\log _{1_{1}}\left(r_{1}\right)\right)^{s_{1}}}{\left(2+\log _{1_{1}}\left(r_{1}\right)\right)^{s_{1}}+\left(\log _{1_{1}}\left(r_{1}\right)\right)^{s_{1}}}, \frac{2\left(\log _{1_{1}}\left(1-h_{1}\right)\right)^{s_{1}}}{\left(2-\log _{1_{1}}\left(1-h_{1}\right)\right)^{s_{1}}+\left(\log _{1_{1}}\left(1-h_{1}\right)\right)^{s_{1}}}\right)
$$

and

$$
s_{2} \rho_{2}=\left(\frac{\left(2+\log _{1_{2}}\left(r_{2}\right)\right)^{s_{2}}-\left(\log _{1_{2}}\left(r_{2}\right)\right)^{s_{2}}}{\left(2+\log _{1_{2}}\left(r_{2}\right)\right)^{s_{2}}+\left(\log _{1_{2}}\left(r_{2}\right)\right)^{s_{2}}}, \frac{2\left(\log _{1_{2}}\left(1-h_{2}\right)\right)^{s_{2}}}{\left(2-\log _{1_{2}}\left(1-h_{2}\right)\right)^{s_{2}}+\left(\log _{1_{2}}\left(1-h_{2}\right)\right)^{s_{2}}}\right)
$$

Then 


$$
\operatorname{LIFEWA}_{s}\left(\rho_{1}, \rho_{2}\right)=\left(\frac{\prod_{j=1}^{2}\left(2-\log _{1_{j}} r_{j}\right)^{s_{j}}-\prod_{j=1}^{2}\left(\log _{1_{j}} r_{j}\right)^{s_{j}}}{\prod_{j=1}^{2}\left(2-\log _{1_{j}} r_{j}\right)^{s_{j}}+\prod_{j=1}^{2}\left(\log _{1_{j}} r_{j}\right)^{s j}}, \frac{2 \prod_{j=1}^{2}\left(\log _{1_{j}}\left(1-h_{j}\right)\right)^{s_{j}}}{\prod_{j=1}^{2}\left(2-\log _{1_{j}}\left(1-h_{j}\right)\right)^{s_{j}}+\prod_{j=1}^{2}\left(\log _{1_{j}}\left(1-h_{j}\right)\right)^{s_{j}}}\right)
$$

Eq. (6) holds for $n=2$, now we show that Eq. (6) is also true for $n=k$, then

$$
\operatorname{LIFEWA}_{s}\left(\rho_{1}, \rho_{2}, \ldots, \rho_{k}\right)=\left(\frac{\prod_{j=1}^{k}\left(2-\log _{1_{j}} r_{j}\right)^{s_{j}}-\prod_{j=1}^{k}\left(\log _{1_{j}} r_{j}\right)^{s_{j}}}{\prod_{j=1}^{k}\left(2-\log _{1_{j}} r_{j}\right)^{s_{j}}+\prod_{j=1}^{k}\left(\log _{1_{j}} r_{j}\right)^{s_{j}}}, \frac{2 \prod_{j=1}^{k}\left(\log _{1_{j}}\left(1-h_{j}\right)\right)^{s_{j}}}{\prod_{j=1}^{k}\left(2-\log _{1_{j}}\left(1-h_{j}\right)\right)^{s_{j}}+\prod_{j=1}^{k}\left(\log _{1_{j}}\left(1-h_{j}\right)\right)^{s_{j}}}\right)
$$

If Eq. (6) is true for $n=k$, then we show that Eq. (6) is true for $n=k+1$, thus

$$
\begin{aligned}
& \operatorname{LIFEWA}_{s}\left(\rho_{1}, \rho_{2}, \ldots, \rho_{k+1}\right) \\
& =\left(\frac{\prod_{j=1}^{k}\left(2-\log _{1_{j}} r_{j}\right)^{s}-\prod_{j=1}^{k}\left(\log _{1_{j}} r_{j}\right)^{s}}{\prod_{j=1}^{k}\left(2-\log _{1_{j}} r_{j}\right)^{s_{j}}+\prod_{j=1}^{k}\left(\log _{1_{j}} r_{j}\right)^{s}}, \frac{2 \prod_{j=1}^{k}\left(\log _{1_{j}}\left(1-h_{j}\right)\right)^{s_{j}}}{\prod_{j=1}^{k}\left(2-\log _{1_{j}}\left(1-h_{j}\right)\right)^{s_{j}}+\prod_{j=1}^{k}\left(\log _{1_{j}}\left(1-h_{j}\right)\right)^{s_{j}}} \oplus_{\varepsilon}\right. \\
& \left(\frac{\left(2-\log _{1_{k+1}} r_{k+1}\right)^{s k+1}-\left(\log _{1_{k+1}} r_{k+1}\right)^{s k+1}}{\left(2-\log _{1_{k+1}} r_{k+1}\right)^{s} k+1+\left(\log _{1_{k+1}} r_{k+1}\right)^{s} k+1}, \frac{2\left(\log _{1_{k+1}}\left(1-h_{k+1}\right)\right)^{s k+1}}{\left(2-\log _{1_{k+1}}\left(1-h_{k+1}\right)\right)^{s} k+1+\left(\log _{1_{k+1}}\left(1-h_{k+1}\right)\right)^{s} k+1}\right)
\end{aligned}
$$

Let $\quad \mathfrak{I}_{1}=\prod_{j=1}^{k}\left(2-\log _{1_{j}} r_{j}\right)^{s_{j}}-\prod_{j=1}^{k}\left(\log _{1_{j}} r_{j}\right)^{s_{j}}, \quad \partial_{1}=\prod_{j=1}^{k}\left(2-\log _{1} r_{j}\right)^{s_{j}}+\prod_{j=1}^{k}\left(\log _{1_{j}} r_{j}\right)^{s_{j}}$

$$
\begin{aligned}
& \mathfrak{I}_{2}=\left(2-\log _{1_{k+1}} r_{k+1}\right)^{s_{k+1}}-\left(\log _{1_{k+1}} r_{k+1}\right)^{s_{k+1}}, \quad \partial_{2}=\left(2-\log _{1_{k+1}} r_{k+1}\right)^{s_{k+1}}+\left(\log _{1_{k+1}} r_{k+1}\right)^{s_{k+1}} \\
& \mathrm{D}_{2}=2\left(\log _{1_{k+1}}\left(1-h_{k+1}\right)\right)^{s_{k+1}}, \quad \mathrm{~h}_{1}=\prod_{j=1}^{k}\left(2-\log _{1_{j}}\left(1-h_{j}\right)\right)^{s_{j}}+\prod_{j=1}^{k}\left(\log _{1_{j}}\left(1-h_{j}\right)\right)^{s_{j}} \\
& \mathrm{~h}_{2}=\left(2-\log _{1_{k+1}}\left(1-h_{k+1}\right)\right)^{s_{k+1}}+\left(\log _{1_{k+1}}\left(1-h_{k+1}\right)\right)^{s}{ }_{k+1}, \mathrm{D}_{1}=2 \prod_{j=1}^{k}\left(\log _{1_{j}}\left(1-h_{j}\right)\right)^{s_{j}}
\end{aligned}
$$

Now putting the above values in Eq. (7), we get the following:

$$
\begin{aligned}
& \operatorname{LIFEWA}_{s}\left(\rho_{1}, \rho_{2}, \ldots, \rho_{k+1}\right) \\
& \begin{aligned}
\left(\frac{\mathfrak{I}_{1}}{\partial_{1}}, \frac{\mathrm{D}_{1}}{\mathrm{~h}_{1}}\right) \oplus_{\varepsilon}\left(\frac{\mathfrak{I}_{2}}{\partial_{2}}, \frac{\mathrm{D}_{2}}{\mathrm{~h}_{2}}\right) & =\left(\frac{\frac{\mathfrak{I}_{1}}{\partial_{1}}+\frac{\mathfrak{I}_{2}}{\partial_{2}}}{1+\left(\frac{\mathfrak{I}_{1}}{\partial_{1}}\right)\left(\frac{\mathfrak{I}_{2}}{\partial_{2}}\right)}, \frac{\frac{\mathrm{D}_{1}}{\mathrm{~h}_{1}} \frac{\mathrm{D}_{2}}{\mathrm{~h}_{2}}}{1+\left(1-\frac{\mathrm{D}_{1}}{\mathrm{~h}_{1}}\right)\left(1-\frac{\mathrm{D}_{2}}{\mathrm{~h}_{2}}\right)}\right) \\
& =\left(\frac{\mathfrak{I}_{1} \partial_{2}+\mathfrak{I}_{2} \partial_{1}}{\partial_{1} \partial_{2}+\mathfrak{I}_{1} \mathfrak{I}_{2}}, \frac{\mathrm{D}_{1} \mathrm{D}_{2}}{2 \mathrm{~h}_{1} \mathrm{~h}_{2}-\mathrm{h}_{1} \mathrm{D}_{2}-\mathrm{D}_{1} \mathrm{~h}_{2}+\mathrm{D}_{1} \mathrm{D}_{2}}\right)
\end{aligned}
\end{aligned}
$$


Now putting $\mathfrak{I}_{1} \partial_{2}+\mathfrak{I}_{2} \partial_{1}, \partial_{1} \partial_{2}+\mathfrak{I}_{1} \mathfrak{I}_{2}, \mathrm{D}_{1} \mathrm{D}_{2}, 2 \mathrm{~h}_{1} \mathrm{~h}_{2}-\mathrm{h}_{1} \mathrm{D}_{2}-\mathrm{D}_{1} \mathrm{~h}_{2}+\mathrm{D}_{1} \mathrm{D}_{2}$, in Eq. (8), we get $\operatorname{LIFEWA}_{s}\left(\rho_{1}, \rho_{2}, \ldots, \rho_{k+1}\right)=\left(\frac{\prod_{j=1}^{k+1}\left(2-\log _{1_{j}} r_{j}\right)^{s_{j}}-\prod_{j=1}^{k+1}\left(\log _{1_{j}} r_{j}\right)^{s_{j}}}{\prod_{j=1}^{k+1}\left(2-\log _{1_{j}} r_{j}\right)^{s_{j}}+\prod_{j=1}^{k+1}\left(\log _{1_{j}} r_{j}\right)^{s_{j}}}, \frac{2 \prod_{j=1}^{k+1}\left(\log _{1_{j}}\left(1-h_{j}\right)\right)^{s_{j}}}{\prod_{j=1}^{k+1}\left(2-\log _{1_{j}}\left(1-h_{j}\right)\right)^{s_{j}}+\prod_{j=1}^{k+1}\left(\log _{1_{j}}\left(1-h_{j}\right)\right)^{s_{j}}}\right)$

Thus, Eq. (8), holds for $n=k+1$, hence Eq. (6), holds for all $n$.

Theorem 6: Let $\rho_{j}=\left(r_{j}, h_{j}\right)$ be a collection of $n$ IFVs, then

$$
\operatorname{LIFEWA}_{s}\left(\rho_{1}, \rho_{2}, \rho_{3}, \ldots, \rho_{n}\right) \leq \operatorname{LIFWA}_{s}\left(\rho_{1}, \rho_{2}, \rho_{3}, \ldots, \rho_{n}\right)
$$

Proof: Using the given information, we get:

$$
\prod_{j=1}^{n}\left(2-\log _{1_{j}} r_{j}\right)^{s_{j}}+\prod_{j=1}^{n}\left(\log _{1_{j}} r_{j}\right)^{s_{j}} \leq \sum_{j=1}^{n} s_{j}\left(2-\log _{1_{j}} r_{j}\right)+\sum_{j=1}^{n} s_{j}\left(\log _{1_{j}} r_{j}\right)
$$

Since

$$
\sum_{j=1}^{n} s_{j}\left(2-\log _{1_{j}} r_{j}\right)+\sum_{j=1}^{n} s_{j}\left(\log _{1_{j}} r_{j}\right)=2
$$

Then

$$
\prod_{j=1}^{n}\left(2-\log _{1} r_{j}\right)^{s_{j}}+\prod_{j=1}^{n}\left(\log _{1} r_{j}\right)^{s_{j}} \leq 2
$$

Thus

$$
\begin{aligned}
\frac{\prod_{j=1}^{n}\left(2-\log _{1_{j}} r_{j}\right)^{s_{j}}-\prod_{j=1}^{n}\left(\log _{1_{j}} r_{j}\right)^{s_{j}}}{\prod_{j=1}^{n}\left(2-\log _{1_{j}} r_{j}\right)^{s_{j}}+\prod_{j=1}^{n}\left(\log _{1_{j}} r_{j}\right)^{s_{j}}} & =1-\frac{2 \prod_{j=1}^{n}\left(\log _{1_{j}} r_{j}\right)^{s_{j}}}{\left(2-\log _{1_{j}} r_{j}\right)^{s_{j}}+\prod_{j=1}^{n}\left(\log _{1_{j}} r_{j}\right)^{s_{j}}} \\
& \leq 1-\prod_{j=1}^{n}\left(\log _{1_{j}} r_{j}\right)^{s_{j}}
\end{aligned}
$$

The equality hold, if $\log _{1} r_{j}$ are equal. Again

$$
\prod_{j=1}^{n}\left(2-\log _{1_{j}}\left(1-h_{j}\right)\right)^{s_{j}}+\prod_{j=1}^{n}\left(\log _{1_{j}}\left(1-h_{j}\right)\right)^{s_{j}} \leq \sum_{j=1}^{n} s_{j}\left(2-\log _{1_{j}}\left(1-h_{j}\right)\right)+\sum_{j=1}^{n} s_{j}\left(\log _{1_{j}}\left(1-h_{j}\right)\right)
$$

As

$$
\sum_{j=1}^{n} s_{j}\left(2-\log _{j}\left(1-h_{j}\right)\right)+\sum_{j=1}^{n} s_{j} \log _{j}\left(1-h_{j}\right)=2
$$


Then

$$
\prod_{j=1}^{n}\left(2-\log _{1_{j}}\left(1-h_{j}\right)\right)^{s_{j}}+\prod_{j=1}^{n}\left(\log _{1_{j}}\left(1-h_{j}\right)\right)^{s_{j}} \leq 2
$$

Thus

$$
\frac{2 \prod_{j=1}^{n}\left(\log _{1_{j}}\left(1-h_{j}\right)\right)^{s_{j}}}{\prod_{j=1}^{n}\left(2-\log _{1_{j}}\left(1-h_{j}\right)\right)^{s_{j}}+\prod_{j=1}^{n}\left(\log _{1_{j}}\left(1-h_{j}\right)\right)^{s_{j}}} \geq \prod_{j=1}^{n}\left(\log _{1_{j}}\left(1-h_{j}\right)\right)^{s_{j}}
$$

The equality hold, if the value of $\log _{1_{j}}\left(1-h_{j}\right)$ are equal:

Let $\operatorname{LIFWA}_{s}\left(\rho_{1}, \rho_{2}, \ldots, \rho_{n}\right)=\rho=\left(r_{\rho}, h_{\rho}\right) \quad$ and $\operatorname{LIFEWA}_{s}\left(\rho_{1}, \rho_{2}, \ldots, \rho_{n}\right)=\rho^{\varepsilon}=\left(r_{\rho^{\varepsilon}}, h_{\rho^{\varepsilon}}\right)$, then Eq. (10), Eq. (11) can be written in the forms: $r_{\rho} \geq r_{\rho^{\varepsilon}}$ and $h_{\rho} \leq h_{\rho^{\varepsilon}}$ respectively. Hence we get: $S(\rho)=r_{\rho}-h_{\rho} \geq r_{\rho^{\varepsilon}}-h_{\rho^{\varepsilon}}=S\left(\rho^{\varepsilon}\right)$. Thus, $S(\rho) \geq S\left(\rho^{\varepsilon}\right)$. Hence, we have two cases:

1): If $S\left(\rho^{\varepsilon}\right)$ p $S(\rho)$, then we have

$$
\operatorname{LIFEWA}_{s}\left(\rho_{1}, \rho_{2}, \rho_{3}, \ldots, \rho_{n}\right) \mathrm{p} \operatorname{LIFWA}_{s}\left(\rho_{1}, \rho_{2}, \rho_{3}, \ldots, \rho_{n}\right)
$$

2): If $S(\rho)=S\left(\rho^{\varepsilon}\right)$, then we get $r_{\rho}=r_{\rho^{\varepsilon}}, h_{\rho}=h_{\rho^{\varepsilon}}$. This implies that $r_{\rho}+h_{\rho}=r_{\rho^{\varepsilon}}+h_{\rho^{\varepsilon}}$. Hence $H(\rho)=H\left(\rho^{\varepsilon}\right)$, thus we get

$$
\operatorname{LIFEWA}_{s}\left(\rho_{1}, \rho_{2}, \rho_{3}, \ldots, \rho_{n}\right)=\operatorname{LIFWA}_{s}\left(\rho_{1}, \rho_{2}, \rho_{3}, \ldots, \rho_{n}\right)
$$

Hence from Eq. (12) and Eq. (13), we get Eq. (9) always true.

Example 1: To develop the above result, we construct an example. For this we consider $\rho_{1}=(0.50,0.30), \rho_{2}=(0.40,0.50), \rho_{3}=(0.50,0.20)$, and $\rho_{4}=(0.30,0.60)$ are four IFNs, $s=(0.10,0.20,0.30,0.40)$, and $1_{1}=1_{2}=1_{3}=1_{4}=0.2$. By applying LIFWA operator, we have

$\operatorname{LIFWA}_{s}\left(\rho_{1}, \rho_{2}, \rho_{3}, \rho_{4}\right)$

$$
\begin{aligned}
& =\left(\begin{array}{l}
1-\left(\log _{0.2} 0.50\right)^{0.10}\left(\log _{0.2} 0.40\right)^{0.20}\left(\log _{0.2} 0.50\right)^{0.30}\left(\log _{0.2} 0.30\right)^{0.40}, \\
\left(\log _{0.2}(1-0.30)\right)^{0.10}\left(\log _{0.2}(1-0.50)\right)^{0.20}\left(\log _{0.2}(1-0.20)\right)^{0.30}\left(\log _{0.2}(1-0.60)\right)^{0.40}
\end{array}\right) \\
& =(0.434,0.319)
\end{aligned}
$$


Now applying LIFEWA operator, we have

$$
\begin{aligned}
& \text { LIFEWA }_{s}\left(\rho_{1}, \rho_{2}, \rho_{3}, \rho_{4}\right) \\
& \begin{array}{l}
\left(\begin{array}{l}
\left(2-\log _{0.2}(0.50)\right)^{0.10}\left(2-\log _{0.2}(0.40)\right)^{0.20}\left(2-\log _{0.2}(0.50)\right)^{0.30}\left(2-\log _{0.2}(0.30)\right)^{0.40} \\
\frac{-\left(\log _{0.2}(0.50)\right)^{0.10}\left(\log _{0.2}(0.40)\right)^{0.20}\left(\log _{0.2}(0.50)\right)^{0.30}\left(\log _{0.2}(0.30)\right)^{0.40}}{\left(2-\log _{0.2}(0.50)\right)^{0.10}\left(2-\log _{0.2}(0.40)\right)^{0.20}\left(2-\log _{0.2}(0.50)\right)^{0.30}\left(2-\log _{0.2}(0.30)\right)^{0.40}}, \\
+\left(\log _{0.2}(0.50)\right)^{0.10}\left(\log _{0.2}(0.40)\right)^{0.20}\left(\log _{0.2}(0.50)\right)^{0.30}\left(\log _{0.2}(0.30)\right)^{0.40}
\end{array}\right. \\
\frac{2\left(\log _{0.2}(1-0.30)\right)^{0.10}\left(\log _{0.2}(1-0.50)\right)^{0.20}\left(\log _{0.2}(1-0.20)\right)^{0.30}\left(\log _{0.2}(1-0.60)\right)^{0.40}}{\left(2-\log _{0.2}(1-0.30)\right)^{0.10}\left(2-\log _{0.2}(1-0.50)\right)^{0.20}\left(2-\log _{0.2}(1-0.20)\right)^{0.30}\left(2-\log _{0.2}(1-0.60)\right)^{0.40}} \\
+\left(\log _{0.2}(1-0.30)\right)^{0.10}\left(\log _{0.2}(1-0.50)\right)^{0.20}\left(\log _{0.2}(1-0.20)\right)^{0.30}\left(\log _{0.2}(1-0.60)\right)^{0.40}
\end{array} \\
& =(0425,0.331)
\end{aligned}
$$

By Definition $3, S($ LIFEWA $)=0.425-0.331=0.094$ and $S($ LIFWA $)=0.434-0.319=0.115$.

Thus from the score function, we get the given result is holds.

Theorem 7: Let $\rho_{j}=\left(r_{j}, h_{j}\right)$ be a collection of $n$ IFNs, then

1) Commutatively: If $\rho_{j}^{*}=\left(r_{j}^{*}, h_{j}^{*}\right)$ be a collection of $n$ IFNs, then

$$
\operatorname{LIFEWA}_{s}\left(\rho_{1}, \rho_{2}, \rho_{3}, \ldots, \rho_{n}\right)=\operatorname{LIFEWA}_{s}\left(\rho_{1}^{*}, \rho_{2}^{*}, \rho_{3}^{*}, \ldots, \rho_{n}^{*}\right)
$$

where $\rho_{j}^{*}$ is any rearrangement of $\rho_{j}$, and $j$ is any positive number.

Proof: Using the given information, we get

$$
\operatorname{LIFEWA}_{s}\left(\rho_{1}, \rho_{2}, \ldots, \rho_{n}\right)=s_{1} \log _{1_{1}} \rho_{1} \oplus_{\varepsilon} s_{2} \log _{1_{2}} \rho_{2} \oplus_{\varepsilon} \ldots \oplus_{\varepsilon} s_{n} \log _{1_{n}} \rho_{n}
$$

and

$$
\operatorname{LIFEWA}_{s}\left(\rho_{1}^{*}, \rho_{2}^{*}, \ldots, \rho_{n}^{*}\right)=s_{1} \log _{1_{1}} \rho_{1}^{*} \oplus_{\varepsilon} s_{2} \log _{1_{2}} \rho_{2}^{*} \oplus_{\varepsilon} \ldots \oplus_{\varepsilon} s_{n} \log _{1_{n}} \rho_{n}^{*}
$$

Since $\rho_{j}^{*}$ is any rearrangement of $\rho_{j}$, thus from Eq. (15) and Eq. (16), we have Eq. (14), is true.

2) Idempotency: If $1_{1}=1_{2}=1_{3}=\ldots=1_{n}=1$, and $\rho_{j}=\rho$, then

$$
\operatorname{LIFEWA}_{s}\left(\rho_{1}, \rho_{2}, \rho_{3}, \ldots, \rho_{n}\right)=\log _{1} \rho
$$

Proof: As we are given that $\rho_{j}=\rho$, then we have 


$$
\begin{aligned}
& \operatorname{LIFEWA}_{s}\left(\rho_{1}, \rho_{2}, \ldots, \rho_{n}\right) \\
& =\left(\frac{\prod_{j=1}^{n}\left(2+\log _{1} r_{j}\right)^{s_{j}}-\prod_{j=1}^{n}\left(\log _{1} r_{j}\right)^{s_{j}}}{\prod_{j=1}^{n}\left(2+\log _{1} r_{j}\right)^{s_{j}}+\prod_{j=1}^{n}\left(\log _{1} r_{j}\right)^{s_{j}}}, \frac{2 \prod_{j=1}^{n}\left(\log _{1}\left(1-h_{j}\right)\right)^{s_{j}}}{\underset{j=1}{\mathrm{C}}\left(2-\log _{1}\left(1-h_{j}\right)\right)^{s_{j}}+\prod_{j=1}^{n}\left(\log _{1}\left(1-h_{j}\right)\right)^{s_{j}}}\right) \\
& =\left(\frac{\prod_{j=1}^{n}\left(2+\log _{1} r\right)^{s_{j}}-\prod_{j=1}^{n}\left(\log _{1} r\right)^{s_{j}}}{\prod_{j=1}^{n}\left(2+\log _{1} r\right)^{s_{j}}+\prod_{j=1}^{n}\left(\log _{1} r\right)^{s_{j}}}, \frac{2 \prod_{j=1}^{n}\left(\log _{1}(1-h)\right)^{s_{j}}}{\underset{j=1}{\mathrm{C}}\left(2-\log _{1}(1-h)\right)^{s_{j}}+\prod_{j=1}^{n}\left(\log _{1}(1-h)\right)^{s_{j}}}\right) \\
& =\left(\frac{\left(2+\log _{1} r\right) \sum_{j=1}^{n} s_{j}-\left(\log _{1} r\right) \sum_{j=1}^{n} s_{j}}{\left(2+\log _{1} r\right) \sum_{j=1}^{n} s_{j}+\left(\log _{1} r\right) \sum_{j=1}^{n} s_{j}}, \frac{2\left(\log _{1}(1-h)\right)_{j=1}^{\sum_{j=1}^{n} s_{j}}}{\left.\left(2-\log _{1}(1-h)\right)\right)_{j=1}^{n} s_{j}+\left(\log _{1}(1-h)\right)_{j=1}^{\sum_{j} s_{j}}}\right) \\
& =\log _{1} \rho
\end{aligned}
$$

3) Boundedness: If $\rho_{\max }=\left(\max _{j}\left\{r_{\rho_{j}}\right\}, \min _{j}\left\{h_{\rho_{j}}\right\}\right), \rho_{\min }=\left(\min _{j}\left\{r_{\rho_{j}}\right\}, \max _{j}\left\{h_{\rho_{j}}\right\}\right)$, then

$$
\log _{1}\left(\rho_{\min }\right) \leq \operatorname{LIFEWA}_{s}\left(\rho_{1}, \rho_{2}, \rho_{3}, \ldots, \rho_{n}\right) \leq \log _{1}\left(\rho_{\max }\right)
$$

Proof: As $\min _{j}\left\{r_{j}\right\} \leq r_{j} \leq \max _{j}\left\{r_{j}\right\}, \min _{j}\left\{h_{j}\right\} \leq h_{j} \leq \max _{j}\left\{h_{j}\right\}$ this means that $\rho_{\min } \leq \rho_{j} \leq \rho_{\max }$. $\operatorname{Let} \operatorname{LIFEWA}\left(\rho_{1}, \ldots, \rho_{n}\right)=\log _{1} \rho, \log _{1} \rho_{\max }=\left(r_{\rho_{\min }}, h_{\rho_{\max }}\right)$ and $\log _{1} \rho_{\min }=\left(r_{\rho_{\max }}, h_{\rho_{\min }}\right)$, then

$$
\begin{aligned}
& r_{\rho}=\frac{\prod_{j=1}^{n}\left(2-\log _{1_{j}} r_{j}\right)^{s}-\prod_{j=1}^{n}\left(\log _{1_{j}} r_{j}\right)^{s} j}{\prod_{j=1}^{n}\left(2-\log _{1_{j}} r_{j}\right)^{s}+\prod_{j=1}^{n}\left(\log _{1_{j}} r_{j}\right)^{s}} \leq \frac{\prod_{j=1}^{n}\left(2-\log _{1_{j}} \max \left\{r_{j}\right\}\right)^{s}{ }^{s}-\prod_{j=1}^{n}\left(\log _{1_{j}} \max \left\{r_{j}\right\}\right)^{s} \prod_{j=1}^{n}\left(2-\log _{1_{j}} \max \left\{r_{j}\right\}\right)^{s}+\prod_{j=1}^{n}\left(\log _{1_{j}} \max \left\{r_{j}\right\}\right)^{s}{ }^{s}}{\prod_{j}} \\
& =\frac{\left(2-\log _{1} \max \left\{r_{j}\right\}\right)-\left(\log _{1} \max \left\{r_{j}\right\}\right)}{\left(2-\log _{1} \max \left\{r_{j}\right\}\right)+\left(\log _{1} \max \left\{r_{j}\right\}\right)}=r_{\rho_{\max }} \\
& r_{\rho}=\frac{\prod_{j=1}^{n}\left(2-\log _{1_{j}} r_{j}\right)^{s}-\prod_{j=1}^{n}\left(\log _{1_{j}} r_{j}\right)^{s}{ }_{\prod_{j=1}}^{n}\left(2-\log _{1_{j}} r_{j}\right)^{s}+\prod_{j=1}^{n}\left(\log _{1_{j}} r_{j}\right)^{s} j}{\left.\prod_{j=1}^{n}\left(2-\log _{1_{j}} \min \left\{r_{j}\right\}\right)^{s}+\prod_{j=1}^{n}\left(\log _{1_{j}} \min \left\{r_{j}\right\}\right)^{s}{ }^{s} \log _{1_{j}} \min \left\{r_{j}\right\}\right)^{s} \prod_{j=1}^{n}\left(\log _{1_{j}} \min \left\{r_{j}\right\}\right)^{s}{ }^{n}} \\
& =\frac{\left(2-\log _{1} \min \left\{r_{j}\right\}\right)-\left(\log _{1} \min \left\{r_{j}\right\}\right)}{\left(2-\log _{1} \min \left\{r_{j}\right\}\right)+\left(\log _{1} \min \left\{r_{j}\right\}\right)}=r_{\rho_{\min }}
\end{aligned}
$$




$$
\begin{aligned}
h_{\rho}=\frac{2 \prod_{j=1}^{n}\left(\log _{1_{j}}\left(1-h_{j}\right)\right)^{s} j}{\prod_{j=1}^{n}\left(2-\log _{1_{j}}\left(1-h_{j}\right)\right)^{s}+\prod_{j=1}^{n}\left(\log _{1_{j}}\left(1-h_{j}\right)\right)^{s} j} & \leq \frac{2 \prod_{j=1}^{n}\left(\log _{1_{j}} \max \left\{1-h_{j}\right\}\right)^{s} j}{\prod_{j=1}^{n}\left(2-\log _{1} \max _{j}\left\{1-h_{j}\right\}\right)^{s}+\prod_{j=1}^{n}\left(\log _{1_{j}} \max \left\{1-h_{j}\right\}\right)^{s} j} \\
& =\frac{2 \log _{1} \max \left\{1-h_{j}\right\}}{\left(2-\log _{1} \max \left\{1-h_{j}\right\}\right)+\log _{1} \max \left\{1-h_{j}\right\}}=h_{\rho_{\max }} \\
h_{\rho}=\frac{2 \prod_{j=1}^{n}\left(\log _{1_{j}}\left(1-h_{j}\right)\right)^{s} j}{\prod_{j=1}^{n}\left(2-\log _{1_{j}}\left(1-h_{j}\right)\right)^{s}+\prod_{j=1}^{n}\left(\log _{1_{j}}\left(1-h_{j}\right)\right)^{s} j} & \geq \frac{2 \prod_{j=1}^{n}\left(\log _{1} \min _{j}\left\{1-h_{j}\right\}\right)^{s} j}{\prod_{j=1}^{n}\left(2-\log _{1_{j}} \min \left\{1-h_{j}\right\}\right)^{s}+\prod_{j=1}^{n}\left(\log _{1} \min _{j}\left\{1-h_{j}\right\}\right)^{s} j} \\
& =\frac{2 \log _{1} \min \left\{1-h_{j}\right\}}{\left(2-\log _{1} \min \left\{1-h_{j}\right\}\right)+\log _{1} \min \left\{1-h_{j}\right\}}=h_{\rho_{\min }}
\end{aligned}
$$

Now by the score function, we get $S\left(\log _{1} \rho\right)=r_{\rho}-h_{\rho} \leq r_{\rho_{\max }}-h_{\rho_{\min }}=S\left(\log _{1} \rho_{\max }\right)$ and $S\left(\log _{1} \rho\right)=r_{\rho}-h_{\rho} \geq r_{\rho_{\min }}-h_{\rho_{\max }}=S\left(\log _{1} \rho_{\min }\right)$. Thus from the score functions, we have $S\left(\log _{1} \rho_{\min }\right) \leq S\left(\log _{1} \rho\right) \leq S\left(\log _{1} \rho_{\max }\right)$. Now we have the following three cases:

i) If $S\left(\log _{1} \rho_{\min }\right) \mathrm{p} S\left(\log _{1} \rho\right) \mathrm{p} S\left(\log _{1} \rho_{\max }\right)$, then we have

$$
\log _{1}\left(\rho_{\min }\right) \mathrm{p} \operatorname{LIFEWA}_{s}\left(\rho_{1}, \rho_{2}, \ldots, \rho_{n}\right) \mathrm{p} \log _{1}\left(\rho_{\max }\right)
$$

ii) If $S\left(\log _{1} \rho\right)=S\left(\log _{1} \rho_{\max }\right)$, this means that $r_{\rho}-h_{\rho}=r_{\rho_{\max }}-h_{\rho_{\min }}$. Hence

$$
r_{\rho}=r_{\rho_{\max }}, h_{\rho}=h_{\rho_{\min }} \text {, this implies that } H\left(\log _{1} \rho\right)=H\left(\log _{1} \rho_{\max }\right) \text {. Thus, we have }
$$

$$
\operatorname{LIFEWA}_{s}\left(\rho_{1}, \rho_{2}, \rho_{3}, \ldots, \rho_{n}\right)=\log _{1}\left(\rho_{\text {max }}\right)
$$

iii) If $S\left(\log _{1} \rho\right)=S\left(\log _{1} \rho_{\min }\right)$, this means that $r_{\rho}-h_{\rho}=r_{\rho_{\min }}-h_{\rho_{\max }}$, this implies that

$$
\begin{gathered}
r_{\rho}=r_{\rho_{\min }} \text { and } h_{\rho}=h_{\rho_{\max }} . \text { Hence } H\left(\log _{1} \rho\right)=H\left(\log _{1} \rho_{\min }\right) \text {. Thus, we have } \\
\operatorname{LIFEWA}_{s}\left(\rho_{1}, \rho_{2}, \rho_{3}, \ldots, \rho_{n}\right)=\log _{1}\left(\rho_{\text {min }}\right)
\end{gathered}
$$

From Eq. (19) to Eq. (21), we have the given Eq. (18) is always holds.

4) Monotonicity: If $\rho_{j}^{*}=\left(r_{j}{ }^{*}, h_{j}{ }^{*}\right)$ be a group of $n$ IFNs, with $r_{j} \leq r_{j}{ }^{*}$ and $h_{j} \geq h_{j}{ }^{*}$, then

$$
\operatorname{LIFEWA}_{s}\left(\rho_{1}, \rho_{2}, \ldots, \rho_{n}\right) \leq \operatorname{LIFEWA}_{s}\left(\rho_{1}{ }^{*}, \rho_{2}{ }^{*}, \ldots, \rho_{n}{ }^{*}\right)
$$

Proof: Proof is similar as above, so it is omitted. 
Definition 11: The LIFEOWA can be defined as:

$\operatorname{LIFEOWA}_{s}\left(\rho_{1}, \rho_{2}, \rho_{3}, \ldots, \rho_{n}\right)$

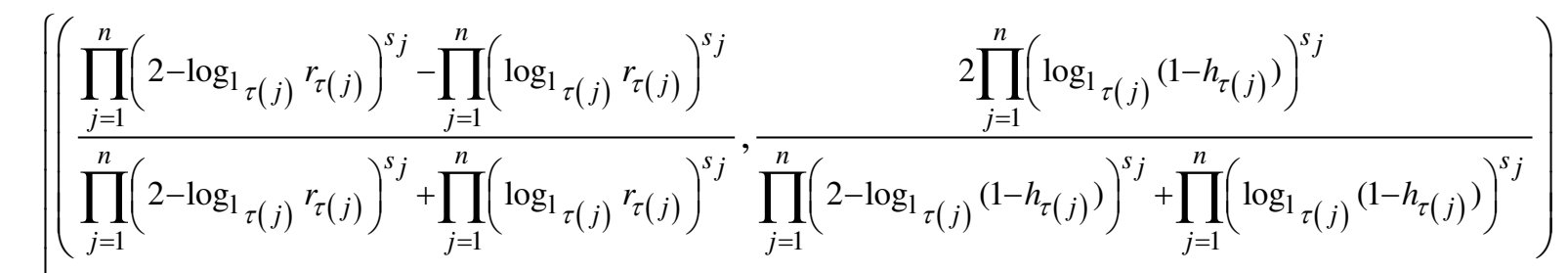

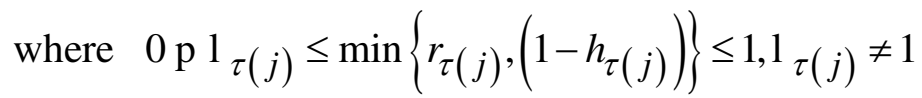

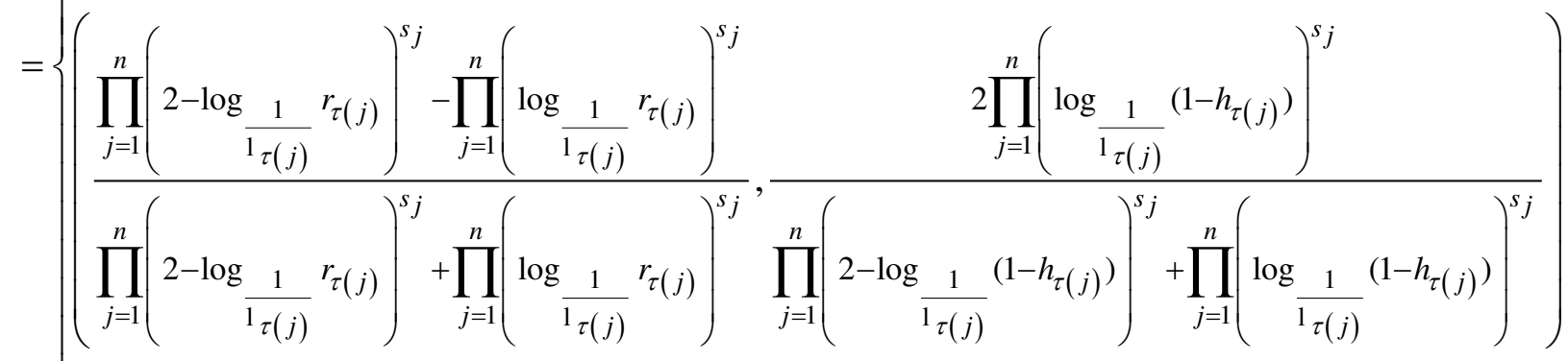

$$
\begin{aligned}
& \text { where } 0 \mathrm{p} \frac{1}{1_{\tau(j)}} \leq \min \left\{r_{\tau(j)},\left(1-h_{\tau(j)}\right)\right\} \leq 1,1_{\tau(j)} \neq 1
\end{aligned}
$$

where $s=\left(s_{1}, s_{2}, \ldots, s_{n}\right)^{T}$ be the weighted vector with condition $s_{j} \in[0,1], \sum_{j=1}^{n} s_{j}=1$, and $(\tau(1), \tau(2), \tau(3), \ldots, \tau(n))$ is any rearrangement of $(1,2,3, \ldots, n)$ such that $\rho_{\tau(j-1)} \geq \rho_{\tau(j)}$.

Example 2: Let $\rho_{1}=(0.40,0.50), \rho_{2}=(0.50,0.30), \rho_{3}=(0.30,0.60)$, and $\rho_{4}=(0.50,0.20)$ are four IFNs, $s=(0.30,0.10,0.20,0.40)$ be their weight vector, and $1_{1}=1_{2}=1_{3}=1_{4}=0.2$. First calculate the score function we get: $S\left(\rho_{1}\right)=(0.40,0.50)=-0.10, S\left(\rho_{2}\right)=(0.50,0.30)=0.20$, $S\left(\rho_{3}\right)=(0.30,0.60)=-0.30, S\left(\rho_{4}\right)=(0.50,0.20)=0.30$. Thus, we get the following: $\rho_{\tau(1)}=(0.50,0.20), \rho_{\tau(2)}=(0.50,0.30), \rho_{\tau(3)}=(0.40,0.50)$, and $\rho_{\tau(4)}=(0.30,0.60)$. Now applying the LIFEOWA operator, we get: 


$$
\begin{aligned}
& \text { LIFEOWA }_{s}\left(\rho_{1}, \rho_{2}, \rho_{3}, \rho_{4}\right) \\
& =\left(\begin{array}{l}
\left(2-\log _{0.2}(0.50)\right)^{0.30}\left(2-\log _{0.2}(0.50)\right)^{0.10}\left(2-\log _{0.2}(0.40)\right)^{0.20}\left(2-\log _{0.2}(0.30)\right)^{0.40} \\
\frac{-\left(\log _{0.2}(0.50)\right)^{0.30}\left(\log _{0.2}(0.50)\right)^{0.10}\left(\log _{0.2}(0.40)\right)^{0.20}\left(\log _{0.2}(0.30)\right)^{0.40}}{\left(2-\log _{0.2}(0.50)\right)^{0.30}\left(2-\log _{0.2}(0.50)\right)^{0.10}\left(2-\log _{0.2}(0.40)\right)^{0.20}\left(2-\log _{0.2}(0.30)\right)^{0.40},} \\
+\left(\log _{0.2}(0.50)\right)^{0.30}\left(\log _{0.2}(0.50)\right)^{0.10}\left(\log _{0.2}(0.40)\right)^{0.20}\left(\log _{0.2}(0.30)\right)^{0.40} \\
\frac{2\left(\log _{0.2}(1-0.20)\right)^{0.30}\left(\log _{0.2}(1-0.30)\right)^{0.10}\left(\log _{0.2}(1-0.50)\right)^{0.20}\left(\log _{0.2}(1-0.60)\right)^{0.40}}{\left(2-\log _{0.2}(1-0.20)\right)^{0.30}\left(2-\log _{0.2}(1-0.30)\right)^{0.10}\left(2-\log _{0.2}(1-0.50)\right)^{0.20}\left(2-\log _{0.2}(1-0.60)\right)^{0.40}} \\
+\left(\log _{0.2}(1-0.20)\right)^{0.30}\left(\log _{0.2}(1-0.30)\right)^{0.10}\left(\log _{0.2}(1-0.50)\right)^{0.20}\left(\log _{0.2}(1-0.60)\right)^{0.40}
\end{array}\right. \\
& = \\
& (043,0.34)
\end{aligned}
$$

Theorem 8: Let $\rho_{j}=\left(r_{j}, h_{j}\right)$ be a group of $n$ IFVs , then their resulting value by using LIFEOWA operator is also IFV.

Proof: Proof is similar to Theorem 5.

Theorem 9: Let $\rho_{j}=\left(r_{j}, h_{j}\right)$ be a group of $n$ IFVs, then

$$
\operatorname{LIFEOWA}_{s}\left(\rho_{1}, \rho_{2}, \rho_{3}, \ldots, \rho_{n}\right) \leq \operatorname{LIFOWA}_{s}\left(\rho_{1}, \rho_{2}, \rho_{3}, \ldots, \rho_{n}\right)
$$

Proof: Proof is similar to Theorem 6 .

Definition 12: The LIFEHA operator can be defined as:

$\operatorname{LIFEHA}_{\gamma, s}\left(\rho_{1}, \rho_{2}, \rho_{2}, \ldots, \rho_{n}\right)$

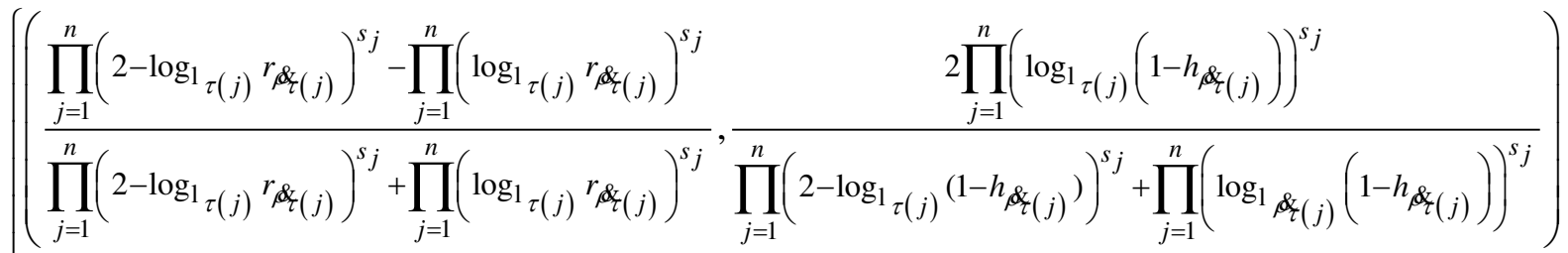

$$
\begin{aligned}
& \text { where } 0 \mathrm{p}_{\tau(j)} \leq \min \left\{r_{\tau(j)},\left(1-h_{\tau(j)}\right)\right\} \leq 1,1_{\tau(j)} \neq 1
\end{aligned}
$$

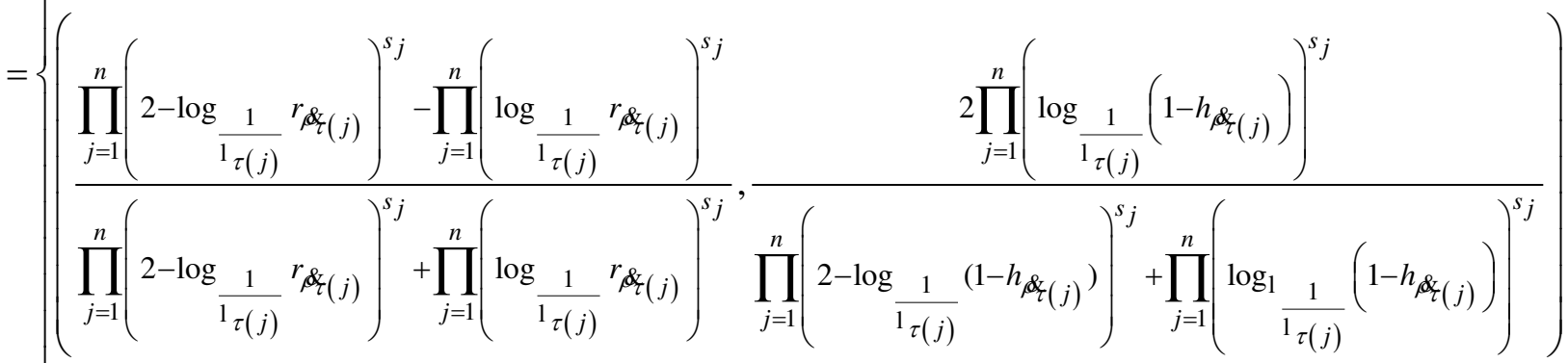

$$
\begin{aligned}
& \text { where } 0 \mathrm{p} \frac{1}{1_{\tau(j)}} \leq \min \left\{r_{\tau(j)},\left(1-h_{\tau(j)}\right)\right\} \leq 1,1_{\tau(j)} \neq 1
\end{aligned}
$$


where $\&_{(j)}$ be the highest WITFVs, $\&_{j}=n \gamma_{j} \rho_{j}$ and $\gamma=\left(\gamma_{1}, \gamma_{2}, \ldots, \gamma_{n}\right)^{T}$ be the weight vector with condition $\gamma_{j} \in[0,1], \sum_{j=1}^{n} \gamma_{j}=1$, and $n$ is constant number. $s=\left(s_{1}, s_{2}, \ldots, s_{n}\right)^{T}$ be the associated vector with conditions $s_{j} \in[0,1]$ and $\sum_{j=1}^{n} s_{j}=1$.

As similar to LIFEWA operator, the LIFEOWA operator and LIFEHA operator also have the same properties such as commutatively, idempotency, boundedness and monotonicity.

Definition 13: The LIFEWG can be defined as:

$$
\begin{aligned}
& \operatorname{LIFEWG}_{s}\left(\rho_{1}, \rho_{2}, \rho_{3}, \ldots, \rho_{n}\right) \\
& \left(\left(\frac{2 \prod_{j=1}^{n}\left(1-\log _{1_{j}} r_{j}\right)^{s j}}{\prod_{j=1}^{n}\left(1+\log _{1_{j}} r_{j}\right)^{s_{j}}+\prod_{j=1}^{n}\left(1-\log _{1_{j}} r_{j}\right)^{s}}, \frac{\prod_{j=1}^{n}\left(1+\log _{1_{j}}\left(1-h_{j}\right)\right)^{s}-\prod_{j=1}^{n}\left(1-\log _{1_{j}}\left(1-h_{j}\right)\right)^{s}}{\prod_{j=1}^{n}\left(1+\log _{1_{j}}\left(1-h_{j}\right)\right)^{s}+\prod_{j=1}^{n}\left(1-\log _{1_{j}}\left(1-h_{j}\right)\right)^{s_{j}}}\right)\right. \\
& \text { where } 0 \mathrm{p}_{j} \leq \min \left\{r_{j},\left(1-h_{j}\right)\right\} \leq 1 ; 1_{j} \neq 1 \\
& =\left\{\left(\frac{2 \prod_{j=1}^{n}\left(1-\log _{\frac{1}{1_{j}}} r_{j}\right)^{s_{j}}}{\prod_{j=1}^{n}\left(1+\log _{\frac{1}{1_{j}}} r_{j}\right)^{s j}+\prod_{j=1}^{n}\left(1-\log _{\frac{1}{1_{j}}} r_{j}\right)^{s}} \frac{\prod_{j=1}^{n}\left(1+\log _{\frac{1}{1_{j}}}\left(1-h_{j}\right)\right)^{s}-\prod_{j=1}^{n}\left(1-\log _{\frac{1}{1_{j}}}\left(1-h_{j}\right)\right)^{s_{j}}}{\prod_{j=1}^{n}\left(1+\log _{\frac{1}{1_{j}}}\left(1-h_{j}\right)\right)^{s j}+\prod_{j=1}^{n}\left(1-\log _{\frac{1}{1_{j}}}\left(1-h_{j}\right)\right)^{s_{j}}}\right)\right. \\
& \text { where } 0 \mathrm{p} \frac{1}{1_{j}} \leq \min \left\{r_{j},\left(1-h_{j}\right)\right\} \leq 1 ; 1_{j} \neq 1
\end{aligned}
$$

where $s=\left(s_{1}, s_{2}, \ldots, s_{n}\right)^{T}$ be the weight vector with conditions $s_{j}$ f 0 and $\sum_{j=1}^{n} s_{j}=1$.

Theorem 10: Let $\rho_{j}=\left(r_{j}, h_{j}\right)$ be a family of $n$ IFVs , then their resulting value by using LIFEWG operator is also a IFV, and

$$
\begin{aligned}
& \operatorname{LIFEWG}_{s}\left(\rho_{1}, \rho_{2}, \rho_{3}, \ldots, \rho_{n}\right) \\
& =\left(\frac{2 \prod_{j=1}^{n}\left(1-\log _{1} r_{j}\right)^{s_{j}}}{\prod_{j=1}^{n}\left(1+\log _{1_{j}} r_{j}\right)^{s_{j}}+\prod_{j=1}^{n}\left(1-\log _{1_{j}} r_{j}\right)^{s j}}, \frac{\prod_{j=1}^{n}\left(1+\log _{1_{j}}\left(1-h_{j}\right)\right)^{s_{j}}-\prod_{j=1}^{n}\left(1-\log _{1_{j}}\left(1-h_{j}\right)\right)^{s_{j}}}{\prod_{j=1}^{n}\left(1+\log _{1_{j}}\left(1-h_{j}\right)\right)^{s_{j}}+\prod_{j=1}^{n}\left(1-\log _{1_{j}}\left(1-h_{j}\right)\right)^{s_{j}}}\right)
\end{aligned}
$$


Proof: This result can be proved by induction formula. For $n=2$

$$
\rho_{1}^{S_{1}}=\left(\frac{\left(1-\log _{1_{1}} r_{1}\right)^{S_{1}}}{\left(1+\log _{1_{1}} r_{1}\right)^{S_{1}}+\left(1-\log _{1_{1}} r_{1}\right)^{S_{1}}}, \frac{\left(1+\log _{1_{1}}\left(1-h_{1}\right)\right)^{S_{1}}-\left(1-\log _{1_{1}}\left(1-h_{1}\right)\right)^{S_{1}}}{\left(1+\log _{1_{1}}\left(1-h_{1}\right)\right)^{S_{1}}+\left(1-\log _{1_{1}}\left(1-h_{1}\right)\right)^{S_{1}}}\right)
$$

and

$$
\rho_{2} s_{2}=\left(\frac{\left(1-\log _{1_{2}} r_{2}\right)^{s_{2}}}{\left(1+\log _{1_{2}} r_{2}\right)^{s_{2}}+\left(1-\log _{1_{2}} r_{2}\right)^{s_{2}}}, \frac{\left(1+\log _{1_{2}}\left(1-h_{2}\right)\right)^{s_{2}}-\left(1-\log _{1_{2}}\left(1-h_{2}\right)\right)^{s_{2}}}{\left(1+\log _{1_{2}}\left(1-h_{2}\right)\right)^{s_{2}}+\left(1-\log _{1_{2}}\left(1-h_{2}\right)\right)^{s_{2}}}\right)
$$

Then

$\operatorname{LIFEWG}_{s}\left(\rho_{1}, \rho_{2}\right)=\left(\frac{2 \prod_{j=1}^{2}\left(1-\log _{1_{j}} r_{j}\right){ }^{s_{j}}}{\prod_{j=1}^{2}\left(1+\log _{1_{j}} r_{j}\right)^{s_{j}}+\prod_{j=1}^{2}\left(1-\log _{1_{j}} r_{j}\right)^{s_{j}}}, \frac{\prod_{j=1}^{2}\left(1+\log _{1_{j}}\left(1-h_{j}\right)\right)^{s_{j}}-\prod_{j=1}^{2}\left(1-\log _{1_{j}}\left(1-h_{j}\right)\right)^{s_{j}}}{\prod_{j=1}^{2}\left(1+\log _{1_{j}}\left(1-h_{j}\right)\right)^{s_{j}}+\prod_{j=1}^{2}\left(1-\log _{1_{j}}\left(1-h_{j}\right)\right)^{s_{j}}}\right)$

For $n=2$, the required result (24) is hold, now we assume that for $n=k$, it is true, then

$$
\operatorname{LIFEWG}_{s}\left(\rho_{1}, \rho_{2}, \ldots, \rho_{k}\right)=\left(\frac{2 \prod_{j=1}^{k}\left(1-\log _{1_{j}} r_{j}\right)^{s_{j}}}{\prod_{j=1}^{k}\left(1+\log _{1_{j}} r_{j}\right)^{s_{j}}+\prod_{j=1}^{k}\left(1-\log _{1_{j}} r_{j}\right)^{s_{j}}} \frac{\prod_{j=1}^{k}\left(1+\log _{j}\left(1-h_{j}\right)\right)^{s_{j}}-\prod_{j=1}^{k}\left(1-\log _{1_{j}}\left(1-h_{j}\right)\right)^{s_{j}}}{\prod_{j=1}^{k}\left(1+\log _{1_{j}}\left(1-h_{j}\right)\right)^{s_{j}}+\prod_{j=1}^{k}\left(1-\log _{1_{j}}\left(1-h_{j}\right)\right)^{s_{j}}}\right)
$$

If the result (24) true for $n=k$, then we want to prove that it is true for $n=k+1$, hence

$$
\begin{aligned}
& \operatorname{LIFEWG}_{s}\left(\rho_{1}, \rho_{2}, \ldots, \rho_{k+1}\right) \\
& =\left(\frac{2 \prod_{j=1}^{k}\left(1-\log _{1_{j}} r_{j}\right)^{s_{j}}}{\prod_{j=1}^{k}\left(1+\log _{1_{j}} r_{j}\right)^{s_{j}}+\prod_{j=1}^{k}\left(1-\log _{1_{j}} r_{j}\right)^{s}}, \frac{\prod_{j=1}^{k}\left(1+\log _{1_{j}}\left(1-h_{j}\right)\right)^{s_{j}}-\prod_{j=1}^{k}\left(1-\log _{1_{j}}\left(1-h_{j}\right)\right)^{s_{j}}}{\prod_{j=1}^{k}\left(1+\log _{1_{j}}\left(1-h_{j}\right)\right)^{s_{j}}+\prod_{j=1}^{k}\left(1-\log _{1_{j}}\left(1-h_{j}\right)\right)^{s_{j}}}\right) \otimes \\
& \left(\frac{2\left(1-\log _{1_{k+1}} r_{k+1}\right)^{s_{k+1}}}{\left(1+\log _{1_{k+1}} r_{k+1}\right)^{s_{k+1}}+\left(1-\log _{1_{k+1}} r_{k+1}\right)^{s_{k+1}}}, \frac{\left(1+\log _{1_{k+1}}\left(1-h_{k+1}\right)\right)^{s_{k+1}}-\left(1-\log _{1_{k+1}}\left(1-h_{k+1}\right)\right)^{s_{k+1}}}{\left(1+\log _{1_{+1}}\left(1-h_{k+1}\right)\right)^{s_{k+1}}+\left(1-\log _{1_{k+1}}\left(1-h_{k+1}\right)\right)^{s k+1}}\right.
\end{aligned}
$$

Let $\partial_{1}=\prod_{j=1}^{k}\left(1+\log _{1_{j}} r_{j}\right)^{s_{j}}+\prod_{j=1}^{k}\left(1-\log _{1_{j}} r_{j}\right)^{s_{j}}, \partial_{2}=\left(1+\log _{1_{k+1}} r_{k+1}\right)^{s_{k+1}}+\left(1-\log _{1_{k+1}} r_{k+1}\right)^{s_{k+1}}$

$$
\mathrm{D}_{1}=\prod_{j=1}^{k}\left(1+\log _{1_{j}}\left(1-h_{j}\right)\right)^{s_{j}}-\prod_{j=1}^{k}\left(1-\log _{1_{j}}\left(1-h_{j}\right)\right)^{s_{j}}, \quad \mathfrak{I}_{2}=2\left(1-\log _{1_{k+1}} r_{k+1}\right)^{s_{k+1}}
$$




$$
\begin{aligned}
& \mathrm{h}_{1}=\prod_{j=1}^{k}\left(1+\log _{1_{j}}\left(1-h_{j}\right)\right)^{s_{j}}+\prod_{j=1}^{k}\left(1-\log _{1_{j}}\left(1-h_{j}\right)\right)^{s_{j}}, \\
& \mathfrak{I}_{1}=2 \prod_{j=1}^{k}\left(1-\log _{1}, r_{j}\right)^{s_{j}} \\
& \mathrm{D}_{2}=\left(1+\log _{1_{k+1}}\left(1-h_{k+1}\right)\right)^{S_{k+1}}-\left(1-\log _{1_{k+1}}\left(1-h_{k+1}\right)\right)^{s_{k+1}} \\
& \mathrm{~h}_{2}=\left(1+\log _{1_{+1}}\left(1-h_{k+1}\right)\right)^{s_{k+1}}+\left(1-\log _{1_{k+1}}\left(1-h_{k+1}\right)\right)^{s_{k+1}}
\end{aligned}
$$

Again putting these values in Eq. (25), we get the following:

$$
\begin{aligned}
\operatorname{LIFEWG}_{s}\left(\rho_{1}, \rho_{2}, \ldots, \rho_{k+1}\right) & \begin{aligned}
\left(\frac{\mathfrak{I}_{1}}{\partial_{1}}, \frac{\mathrm{D}_{1}}{\mathrm{~h}_{1}}\right) \otimes_{\varepsilon}\left(\frac{\mathfrak{I}_{2}}{\partial_{2}}, \frac{\mathrm{D}_{2}}{\mathrm{~h}_{2}}\right) & =\left(\frac{\frac{\mathfrak{I}_{1}}{\partial_{1}} \frac{\mathfrak{I}_{2}}{\partial_{2}}}{1+\left(1-\frac{\mathfrak{I}_{1}}{\partial_{1}}\right)\left(1-\frac{\mathfrak{I}_{2}}{\partial_{2}}\right)}, \frac{\frac{\mathrm{D}_{1}}{\mathrm{~h}_{1}}+\frac{\mathrm{D}_{2}}{\mathrm{~h}_{2}}}{1+\left(\frac{\mathrm{D}_{1}}{\mathrm{~h}_{1}}\right)\left(\frac{\mathrm{D}_{2}}{\mathrm{~h}_{2}}\right)}\right) \\
& =\left(\frac{\mathfrak{I}_{1} \mathfrak{I}_{2}}{2 \partial_{1} \partial_{2}-\partial_{1} \mathfrak{I}_{2}-\mathfrak{I}_{1} \partial_{2}+\mathfrak{I}_{1} \mathfrak{I}_{2}}, \frac{\mathrm{D}_{1} \mathrm{~h}_{2}+\mathrm{D}_{2} \mathrm{~h}_{1}}{\mathrm{~h}_{1} \mathrm{~h}_{2}+\mathrm{D}_{1} \mathrm{D}_{2}}\right)
\end{aligned}
\end{aligned}
$$

Putting $\mathfrak{I}_{1} \mathfrak{I}_{2}, 2 \partial_{1} \partial_{2}-\partial_{1} \mathfrak{I}_{2}-\mathfrak{I}_{1} \partial_{2}+\mathfrak{I}_{1} \mathfrak{I}_{2}, \mathrm{D}_{1} \mathrm{~h}_{2}+\mathrm{D}_{2} \mathrm{~h}_{1}, \mathrm{~h}_{1} \mathrm{~h}_{2}+\mathrm{D}_{1} \mathrm{D}_{2}$ in Eq. (26), we get

$$
\operatorname{LIFEWG}_{s}\left(\rho_{1}, \ldots, \rho_{k+1}\right)=\left(\frac{2 \prod_{j=1}^{k+1}\left(1-\log _{1} r_{j}\right)^{s_{j}}}{\prod_{j=1}^{k+1}\left(1+\log _{1_{j}} r_{j}\right)^{s_{j}}+\prod_{j=1}^{k+1}\left(1-\log _{1_{j}} r_{j}\right)^{s j}}, \frac{\prod_{j=1}^{k+1}\left(1+\log _{1_{j}}\left(1-h_{j}\right)\right)^{s}-\prod_{j=1}^{k+1}\left(1-\log _{1_{j}}\left(1-h_{j}\right)\right)^{s_{j}}}{\prod_{j=1}^{k+1}\left(1+\log _{1_{j}}\left(1-h_{j}\right)\right)^{s_{j}}+\prod_{j=1}^{k+1}\left(1-\log _{1_{j}}\left(1-h_{j}\right)\right)^{s_{j}}}\right)
$$

For $n=k+1$, the given Eq. (24) holds. Thus the give result is true for all $n$.

Theorem 11: Let $\rho_{j}=\left(r_{j}, h_{j}\right)$ be a family of $n$ IFVs, then

$$
\operatorname{LIFWG}_{s}\left(\rho_{1}, \rho_{2}, \ldots, \rho_{n}\right) \leq \operatorname{LIFEWG}_{s}\left(\rho_{1}, \rho_{2}, \ldots, \rho_{n}\right)
$$

Proof: Using the given information, then we get:

$$
\prod_{j=1}^{n}\left(1+\log _{1_{j}} r_{j}\right)^{s_{j}}+\prod_{j=1}^{n}\left(1-\log _{1_{j}} r_{j}\right)^{s_{j}} \leq \sum_{j=1}^{n} s_{j}\left(1+\log _{1_{j}} r_{j}\right)+\sum_{j=1}^{n} s_{j}\left(1-\log _{1_{j}} r_{j}\right)
$$

As

$$
\sum_{j=1}^{n} s_{j}\left(1+\log _{1_{j}} r_{j}\right)+\sum_{j=1}^{n} s_{j}\left(1-\log _{1_{j}} r_{j}\right)=2
$$

and

$$
\prod_{j=1}^{n}\left(1+\log _{1} r_{j}\right)^{s_{j}}+\prod_{j=1}^{n}\left(1-\log _{1_{j}} r_{j}\right)^{s_{j}} \leq 2
$$


Thus

$$
\frac{2 \prod_{j=1}^{n}\left(1-\log _{1_{j}} r_{j}\right)^{s_{j}}}{\prod_{j=1}^{n}\left(1+\log _{1_{j}} r_{j}\right)^{s_{j}}+\prod_{j=1}^{n}\left(1-\log _{1_{j}} r_{j}\right)^{s_{j}}} \geq \prod_{j=1}^{n}\left(1-\log _{1_{j}} r_{j}\right)^{s_{j}}
$$

The quality holds, if and only if $\log _{1_{j}} r_{j}$ are equal. Again

$\prod_{j=1}^{n}\left(1+\log _{1_{j}}\left(1-h_{j}\right)\right)^{s_{j}}+\prod_{j=1}^{n}\left(1-\log _{1_{j}}\left(1-h_{j}\right)\right)^{s_{j}} \leq \sum_{j=1}^{n} s_{j}\left(1+\log _{1_{j}}\left(1-h_{j}\right)\right)+\sum_{j=1}^{n} s_{j}\left(1-\log _{1_{j}}\left(1-h_{j}\right)\right)$

Since

$$
\sum_{j=1}^{n} s_{j}\left(1+\log _{1_{j}}\left(1-h_{j}\right)\right)+\sum_{j=1}^{n} s_{j}\left(1-\log _{1_{j}}\left(1-h_{j}\right)\right)=2
$$

Then

$$
\prod_{j=1}^{n}\left(1+\log _{1_{j}}\left(1-h_{j}\right)\right)^{s_{j}}+\prod_{j=1}^{n}\left(1-\log _{1_{j}}\left(1-h_{j}\right)\right)^{s_{j}} \leq 2
$$

Thus

$$
\frac{\prod_{j=1}^{n}\left(1+\log _{1_{j}}\left(1-h_{j}\right)\right)^{s_{j}}-\prod_{j=1}^{n}\left(1-\log _{1_{j}}\left(1-h_{j}\right)\right)^{s_{j}}}{\prod_{j=1}^{n}\left(1+\log _{1_{j}}\left(1-h_{j}\right)\right)^{s_{j}}+\prod_{j=1}^{n}\left(1-\log _{1_{j}}\left(1-h_{j}\right)\right)^{s_{j}}} \leq 1-\prod_{j=1}^{n}\left(1-\log _{1_{j}}\left(1-h_{j}\right)\right)^{s_{j}}
$$

The quality holds if $\log _{1}\left(1-h_{j}\right)$ are equal. Again let LIFWG ${ }_{s}\left(\rho_{1}, \rho_{2}, \ldots, \rho_{n}\right)=\rho=\left(r_{\rho}, h_{\rho}\right)$ and LIFEWG ${ }_{s}\left(\rho_{1}, \rho_{2}, \ldots, \rho_{n}\right)=\rho^{\varepsilon}=\left(r_{\rho^{\varepsilon}}, h_{\rho^{\varepsilon}}\right)$.Then Eq. (28) and Eq. (29) can be written in the new forms such as, $r_{\rho} \leq r_{\rho^{\varepsilon}}$ and $h_{\rho} \geq h_{\rho^{\varepsilon}}$ respectively. Then, we have $S(\rho)=r_{\rho}-h_{\rho} \leq r_{\rho^{\varepsilon}}-h_{\rho^{\varepsilon}}=S\left(\rho^{\varepsilon}\right)$, hence $S(\rho) \leq S\left(\rho^{\varepsilon}\right)$. Thus from the above relations we get the following two cases:

Case 1: If $S(\rho) \mathrm{p} S\left(\rho^{\varepsilon}\right)$, then we have

$$
\operatorname{LIFWG}_{s}\left(\rho_{1}, \rho_{2}, \ldots, \rho_{n}\right) \mathrm{p} \mathrm{LIFEWG}\left(\rho_{1}, \rho_{2}, \ldots, \rho_{n}\right)
$$


Case 2: If $S(\rho)=S\left(\rho^{\varepsilon}\right)$, this means that $r_{\rho}=r_{\rho^{\varepsilon}}$ and $h_{\rho}=h_{\rho^{\varepsilon}}$. We have $r_{\rho}+h_{\rho}=r_{\rho^{\varepsilon}}+h_{\rho^{\varepsilon}}$ and hence $H(\rho)=H\left(\rho^{\varepsilon}\right)$. Thus we get

$$
\operatorname{LIFWG}_{s}\left(\rho_{1}, \rho_{2}, \ldots, \rho_{n}\right)=\operatorname{LIFEWG}_{s}\left(\rho_{1}, \rho_{2}, \ldots, \rho_{n}\right)
$$

From the Eq. (30) and Eq. (31), we get the result (27) always true.

Example 3: Let $\rho_{1}=(0.30,0.50), \rho_{2}=(0.50,0.40), \rho_{3}=(0.50,0.50)$ and $\rho_{4}=(0.60,0.30)$ are IFNs, $s=(0.40,0.30,0.20,0.10)$ and $1_{1}=1_{2}=1_{3}=1_{4}=0.2$. By LIFEWG operator, we have

$$
\begin{aligned}
& \mathrm{LIFEWG}_{s}\left(\rho_{1}, \rho_{2}, \rho_{3}, \rho_{4}\right) \\
& \left(\frac{2\left(1-\log _{0.2}(0.30)\right)^{0.40}\left(1-\log _{0.2}(0.50)\right)^{0.30}\left(1-\log _{0.2}(0.50)\right)^{0.20}\left(1-\log _{0.2}(0.60)\right)^{0.10}}{\left(1+\log _{0.2}(0.30)\right)^{0.40}\left(1+\log _{0.2}(0.50)\right)^{0.30}\left(1+\log _{0.2}(0.50)\right)^{0.20}\left(1+\log _{0.2}(0.60)\right)^{0.10}},\right. \\
& =\begin{array}{l}
+\left(1-\log _{0.2}(0.30)\right)^{0.40}\left(1-\log _{0.2}(0.50)\right)^{0.30}\left(1-\log _{0.2}(0.50)\right)^{0.20}\left(1-\log _{0.2}(0.60)\right)^{0.10} \\
\left(1+\log _{0.2}(1-0.50)\right)^{0.40}\left(1+\log _{0.2}(1-0.40)\right)^{0.30}\left(1+\log _{0.2}(1-0.50)\right)^{0.20}\left(1+\log _{0.2}(1-0.30)\right)^{0.10}
\end{array} \\
& \frac{-\left(1-\log _{0.2}(1-0.50)\right)^{0.40}\left(1-\log _{0.2}(1-0.40)\right)^{0.30}\left(1-\log _{0.2}(1-0.50)\right)^{0.20}\left(1-\log _{0.2}(1-0.30)\right)^{0.10}}{\left(1+\log _{0.2}(1-0.50)\right)^{0.40}\left(1+\log _{0.2}(1-0.40)\right)^{0.30}\left(1+\log _{0.2}(1-0.50)\right)^{0.20}\left(1+\log _{0.2}(1-0.30)\right)^{0.10}} \\
& \left.+\left(1-\log _{0.2}(1-0.50)\right)^{0.40}\left(1-\log _{0.2}(1-0.40)\right)^{0.30}\left(1-\log _{0.2}(1-0.50)\right)^{0.20}\left(1-\log _{0.2}(1-0.30)\right)^{0.10}\right) \\
& =(0.427,0.384)
\end{aligned}
$$

Now using LIFWG operator, we have

$$
\begin{aligned}
& \operatorname{LIFWG}_{S}\left(\rho_{1}, \rho_{2}, \rho_{3}, \rho_{4}\right) \\
& =\left(\begin{array}{l}
\left(1-\log _{0.2}(0.30)\right)^{0.40}\left(1-\log _{0.2}(0.50)\right)^{0.30}\left(1-\log _{0.2}(0.50)\right)^{0.20}\left(1-\log _{0.2}(0.60)\right)^{0.10}, \\
1-\left(1-\log _{0.2}(1-0.50)\right)^{0.40}\left(1-\log _{0.2}(1-0.40)\right)^{0.30}\left(1-\log _{0.2}(1-0.50)\right)^{0.20}\left(1-\log _{0.2}(1-0.30)\right)^{0.10}
\end{array}\right) \\
& =(0.417,0.380)
\end{aligned}
$$

By Definition 3, $S($ LIFEWG $)=0.427-0.384=0.043$ and $S($ LIFWG $)=0.417-0.380=0.037$.

Thus from the score function, we get the given result is holds.

Theorem 12: Let $\rho_{j}=\left(r_{j}, h_{j}\right)$ be a group of $n$ IFNs, then

1) Commutatively: If $\rho_{j}^{*}=\left(r_{j}^{*}, h_{j}^{*}\right)$ be a group of $n$ IFNs, then

$$
\operatorname{LIFEWG}_{s}\left(\rho_{1}, \rho_{2}, \ldots, \rho_{n}\right)=\operatorname{LIFEWG}_{s}\left(\rho_{1}^{*}, \rho_{2}^{*}, \ldots, \rho_{n}^{*}\right)
$$

where $\rho_{j}^{*}$ is any rearrangement of $\rho_{j}$, and $j$ is any positive number.

Proof: As we know that 


$$
\operatorname{LIFEWG}_{s}\left(\rho_{1}, \rho_{2}, \ldots, \rho_{n}\right)=\left(\log _{1_{1}} \rho_{1}\right)^{s_{1}} \otimes_{\varepsilon}\left(\log _{1_{2}} \rho_{2}\right)^{s_{2}} \otimes_{\varepsilon} \ldots \otimes_{\varepsilon}\left(\log _{1_{n}} \rho_{n}\right)^{s_{n}}
$$

and

$$
\operatorname{LIFEWG}_{s}\left(\rho_{1}^{*}, \rho_{2}^{*}, \ldots, \rho_{n}^{*}\right)=\left(\log _{1_{1}} \rho_{1}^{*}\right)^{S_{1}} \otimes_{\varepsilon}\left(\log _{1_{2}} \rho_{2}^{*}\right)^{S_{2}} \otimes_{\varepsilon} \ldots \otimes_{\varepsilon}\left(\log _{1_{n}} \rho_{n}^{*}\right)^{s_{n}}
$$

Since $\rho_{j}^{*}$ is any rearrangement of $\rho_{j}$. Thus from Eq. (33) and Eq. (34), we have Eq. (32), holds.

2) Idempotency: If $\rho_{j}=\rho$ for all $j$, and $1_{1}=1_{2}=1_{3}=\ldots=1_{n}=1$, then

$$
\operatorname{LIFEWG}_{s}\left(\rho_{1}, \rho_{2}, \rho_{3}, \ldots, \rho_{n}\right)=\log _{1} \rho
$$

Proof: Since $\rho_{j}=\rho$ for all $j$, then we have

$$
\begin{aligned}
& \operatorname{LIFEWG}_{s}\left(\rho_{1}, \rho_{2}, \rho_{3}, \ldots, \rho_{n}\right) \\
& =\left(\frac{2 \prod_{j=1}^{n}\left(1-\log _{1} r_{j}\right)^{s_{j}}}{\prod_{j=1}^{n}\left(1+\log _{1} r_{j}\right)^{s_{j}}+\prod_{j=1}^{n}\left(1-\log _{1} r_{j}\right)^{s_{j}}}, \frac{\prod_{j=1}^{n}\left(1+\log _{1}\left(1-h_{j}\right)\right)^{s_{j}}-\prod_{j=1}^{n}\left(1-\log _{1}\left(1-h_{j}\right)\right)^{s_{j}}}{\prod_{j=1}^{n}\left(1+\log _{1}\left(1-h_{j}\right)\right)^{s_{j}}+\prod_{j=1}^{n}\left(1-\log _{1}\left(1-h_{j}\right)\right)^{s_{j}}}\right) \\
& =\left(\frac{2\left(1-\log _{1} r\right)_{j=1}^{n} s_{j}}{\left(1+\log _{1} r\right)_{j=1}^{n} s_{j}+\left(1-\log _{1} r\right) \sum_{j=1}^{n} s_{j}}, \frac{\left(1+\log _{1}(1-h)\right)_{j=1}^{n} s_{j}-\left(1-\log _{1}(1-h)\right)_{j=1}^{n} s_{j}}{\left(1+\log _{1}(1-h)\right)^{s_{j}}+\left(1-\log _{1}(1-h)\right)_{j=1}^{n} s_{j}}\right) \\
& =\left(\frac{2\left(1-\log _{1} r\right)}{\left(1+\log _{1} r\right)+\left(1-\log _{1} r\right)}, \frac{\left(1+\log _{1}(1-h)\right)-\left(1-\log _{1}(1-h)\right)}{\left(1+\log _{1}(1-h)\right)+\left(1-\log _{1}(1-h)\right)}\right)=\log _{1} \rho
\end{aligned}
$$

3) Boundedness: If $\rho_{\max }=\left(\max _{j}\left\{r_{\rho_{j}}\right\}, \min _{j}\left\{h_{\rho_{j}}\right\}\right), \rho_{\min }=\left(\min _{j}\left\{r_{\rho_{j}}\right\}, \max _{j}\left\{h_{\rho_{j}}\right\}\right)$, then

$$
\log _{1}\left(\rho_{\text {min }}\right) \leq \operatorname{LIFEWG}_{s}\left(\rho_{1}, \rho_{2}, \rho_{3}, \ldots, \rho_{n}\right) \leq \log _{1}\left(\rho_{\text {max }}\right)
$$

Proof: Since $\min _{j}\left\{r_{j}\right\} \leq r_{j} \leq \max _{j}\left\{r_{j}\right\}, \min _{j}\left\{h_{j}\right\} \leq h_{j} \leq \max _{j}\left\{h_{j}\right\}$, this means $\rho_{\min } \leq \rho_{j} \leq \rho_{\max }$. Let LIFEWG $\left(\rho_{1}, \ldots, \rho_{n}\right)=\log _{1} \rho=\left(r_{\rho}, h_{\rho}\right), \log _{1} \rho_{\max }=\left(r_{\rho_{\min }}, h_{\rho_{\max }}\right), \quad \log _{1} \rho_{\min }=\left(r_{\rho_{\max }}, h_{\rho_{\min }}\right)$. Thus we get the following relation: 


$$
\begin{aligned}
& r_{\rho}=\frac{2 \prod_{j=1}^{n}\left(1-\log _{1_{j}} r_{j}\right)^{s j}}{\prod_{j=1}^{n}\left(1+\log _{1_{j}} r_{j}\right)^{s_{j}}+\prod_{j=1}^{n}\left(1-\log _{1_{j}} r_{j}\right)^{s j}} \leq \frac{2 \prod_{j=1}^{n}\left(1-\log _{1_{j}} \max \left\{r_{j}\right\}\right)^{s_{j}}}{\prod_{j=1}^{n}\left(1+\log _{1_{j}} \max \left\{r_{j}\right\}\right)^{s_{j}}+\prod_{j=1}^{n}\left(1-\log _{1_{j}} \max \left\{r_{j}\right\}\right)^{s_{j}}} \\
& =\frac{2\left(1-\log _{1} \max \left\{r_{j}\right\}\right)^{s_{j}}}{\left(1+\log _{1} \max \left\{r_{j}\right\}\right)^{s_{j}}+\left(1-\log _{1} \max \left\{r_{j}\right\}\right)^{s_{j}}}=r_{\rho_{\max }} \\
& r_{\rho}=\frac{2 \prod_{j=1}^{n}\left(1-\log _{1_{j}} r_{j}\right)^{s_{j}}}{\prod_{j=1}^{n}\left(1+\log _{1_{j}} r_{j}\right)^{s_{j}}+\prod_{j=1}^{n}\left(1-\log _{1_{j}} r_{j}\right)^{s_{j}}} \geq \frac{2 \prod_{j=1}^{n}\left(1-\log _{1_{j}} \min \left\{r_{j}\right\}\right)^{s_{j}}}{\prod_{j=1}^{n}\left(1+\log _{1_{j}} \min \left\{r_{j}\right\}\right)^{s_{j}}+\prod_{j=1}^{n}\left(1-\log _{1_{j}} \min \left\{r_{j}\right\}\right)^{s_{j}}} \\
& =\frac{2\left(1-\log _{1} \min \left\{r_{j}\right\}\right)^{s_{j}}}{\left(1+\log _{1} \min \left\{r_{j}\right\}\right)^{s_{j}}+\left(1-\log _{1} \min \left\{r_{j}\right\}\right)^{s_{j}}}=r_{\rho_{\min }} \\
& h_{\rho}=\frac{\prod_{j=1}^{n}\left(1+\log _{1_{j}}\left(1-h_{j}\right)\right)^{s j}-\prod_{j=1}^{n}\left(1-\log _{1_{j}}\left(1-h_{j}\right)\right)^{s}}{\prod_{j=1}^{n}\left(1+\log _{1_{j}}\left(1-h_{j}\right)\right)^{s_{j}}+\prod_{j=1}^{n}\left(1-\log _{1_{j}}\left(1-h_{j}\right)\right)^{s j}} \leq \frac{\prod_{j=1}^{n}\left(1+\log _{1_{j}} \max \left\{1-h_{j}\right\}\right)^{s_{j}}-\prod_{j=1}^{n}\left(1-\log _{1_{j}} \max \left\{1-h_{j}\right\}\right)^{s_{j}}}{\prod_{j=1}^{n}\left(1+\log _{1_{j}} \max \left\{1-h_{j}\right\}\right)^{s_{j}}+\prod_{j=1}^{n}\left(1-\log _{1_{j}} \max \left\{1-h_{j}\right\}\right)^{s_{j}}} \\
& =\frac{\left(1+\log _{1} \max \left\{1-h_{j}\right\}\right)^{s_{j}}-\left(1-\log _{1} \max \left\{1-h_{j}\right\}\right)^{s_{j}}}{\left(1+\log _{1} \max \left\{1-h_{j}\right\}\right)^{s_{j}}+\left(1-\log _{1} \max \left\{1-h_{j}\right\}\right)^{s_{j}}}=h_{\rho_{\max }} \\
& h_{\rho}=\frac{\prod_{j=1}^{n}\left(1+\log _{1_{j}}\left(1-h_{j}\right)\right)^{s_{j}}-\prod_{j=1}^{n}\left(1-\log _{1_{j}}\left(1-h_{j}\right)\right)^{s}}{\prod_{j=1}^{n}\left(1+\log _{1_{j}}\left(1-h_{j}\right)\right)^{s_{j}}+\prod_{j=1}^{n}\left(1-\log _{1_{j}}\left(1-h_{j}\right)\right)^{s_{j}}} \geq \frac{\prod_{j=1}^{n}\left(1+\log _{1_{j}} \min \left\{1-h_{j}\right\}\right)^{s}-\prod_{j=1}^{n}\left(1-\log _{1_{j}} \min \left\{1-h_{j}\right\}\right)^{s_{j}}}{\prod_{j=1}^{n}\left(1+\log _{1_{j}} \min \left\{1-h_{j}\right\}\right)^{s_{j}}+\prod_{j=1}^{n}\left(1-\log _{1_{j}} \min \left\{1-h_{j}\right\}\right)^{s_{j}}} \\
& =\frac{\left(1+\log _{1} \min \left\{1-h_{j}\right\}\right)^{s_{j}}-\left(1-\log _{1} \min \left\{1-h_{j}\right\}\right)^{s_{j}}}{\left(1+\log _{1} \min \left\{1-h_{j}\right\}\right)^{s_{j}}+\left(1-\log _{1} \min \left\{1-h_{j}\right\}\right)^{s_{j}}}=h_{\rho_{\min }}
\end{aligned}
$$

By the score function, we have $S\left(\log _{1} \rho\right)=r_{\rho}-h_{\rho} \leq r_{\rho_{\max }}-h_{\rho_{\min }}=S\left(\log _{1} \rho_{\max }\right)$ and $S\left(\log _{1} \rho\right)=r_{\rho}-h_{\rho} \geq r_{\rho_{\min }}-h_{\rho_{\max }}=S\left(\log _{1} \rho_{\min }\right) . \quad$ So $S\left(\log _{1} \rho_{\min }\right) \leq S\left(\log _{1} \rho\right) \leq S\left(\log _{1} \rho_{\max }\right)$.

Now we have the following three cases:

i) If $S\left(\log _{1} \rho_{\min }\right) \mathrm{p} S\left(\log _{1} \rho\right) \mathrm{p} S\left(\log _{1} \rho_{\max }\right)$, then

$$
\log _{1}\left(\rho_{\min }\right) \mathrm{p} \mathrm{LIFEWG}\left(\rho_{1}, \rho_{2}, \rho_{3}, \ldots, \rho_{n}\right) \mathrm{p} \log _{1}\left(\rho_{\max }\right)
$$


ii) $\quad S\left(\log _{1} \rho\right)=S\left(\log _{1} \rho_{\max }\right)$, this means that $r_{\rho}-h_{\rho}=r_{\rho_{\max }}-h_{\rho_{\min }}$ and hence $r_{\rho}=r_{\rho_{\max }}$ and $h_{\rho}=h_{\rho_{\min }}$. Hence $H\left(\log _{1} \rho\right)=H\left(\log _{1} \rho_{\max }\right)$. Thus we have

$$
\operatorname{LIFEWG}_{s}\left(\rho_{1}, \rho_{2}, \rho_{3}, \ldots, \rho_{n}\right)=\log _{1}\left(\rho_{\max }\right)
$$

iii) $\quad S\left(\log _{1} \rho\right)=S\left(\log _{1} \rho_{\min }\right)$, this means that $r_{\rho}-h_{\rho}=r_{\rho_{\min }}-h_{\rho_{\max }}$ and hence $r_{\rho}=r_{\rho_{\min }}$ and $h_{\rho}=h_{\rho_{\max }}$. Hence $H\left(\log _{1} \rho\right)=H\left(\log _{1} \rho_{\min }\right)$. Thus we have

$$
\operatorname{LIFEWG}_{s}\left(\rho_{1}, \rho_{2}, \rho_{2}, \ldots, \rho_{n}\right)=\log _{1}\left(\rho_{\min }\right)
$$

From Eq. (37) to Eq. (39) the given result Eq. (36) is true.

4) Monotonicity: If $\rho_{j}^{*}=\left(r_{j}^{*}, h_{j}^{*}\right)$ be a group of $n$ IFNs such as, $r_{j} \leq r_{j}^{*}, h_{j} \geq h_{j}{ }^{*}$, then

$$
\operatorname{LIFEWG}_{s}\left(\rho_{1}, \rho_{2}, \ldots, \rho_{n}\right) \leq \operatorname{LIFEWG}_{s}\left(\rho_{1}^{*}, \rho_{2}^{*}, \ldots, \rho_{n}^{*}\right)
$$

Proof: The proof is parallel as above, so it is omitted.

Definition 14: The LIFEOWG can be defined as:

$\operatorname{LIFEOWG}_{s}\left(\rho_{1}, \rho_{2}, \rho_{3}, \ldots, \rho_{n}\right)$

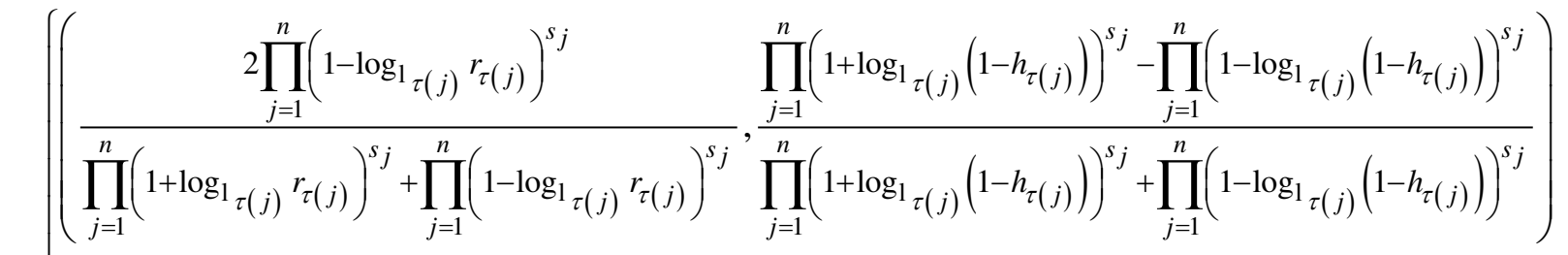

$$
\begin{aligned}
& \text { where } 0 \mathrm{p} 1_{\tau(j)} \leq \min \left\{r_{\tau(j)},\left(1-h_{\tau(j)}\right)\right\} \leq 1 ; 1_{\tau(j)} \neq 1
\end{aligned}
$$

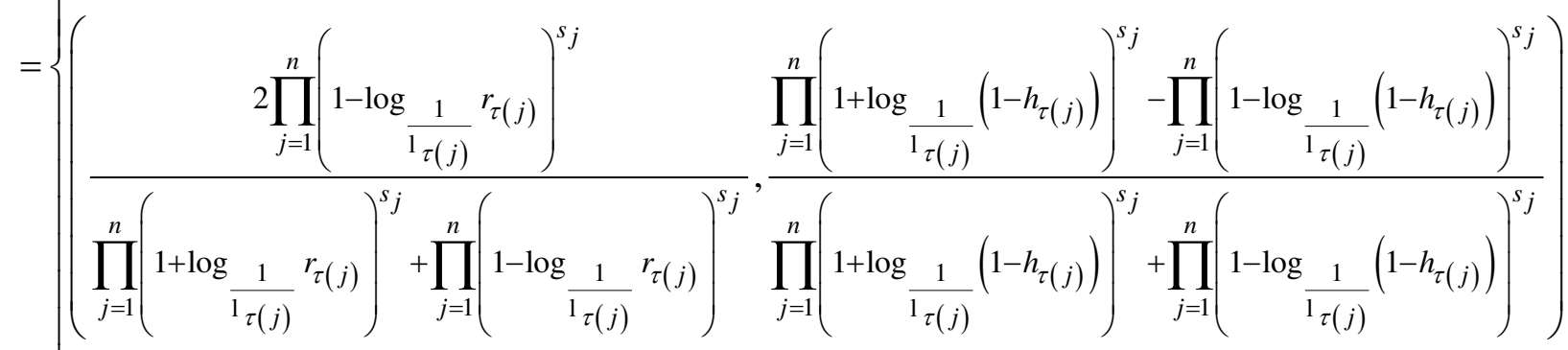

$$
\begin{aligned}
& \text { where } 0 \mathrm{p} \frac{1}{1_{\tau(j)}} \leq \min \left\{r_{\tau(j)},\left(1-h_{\tau(j)}\right)\right\} \leq 1 ; 1_{\tau(j)} \neq 1
\end{aligned}
$$

where $s=\left(s_{1}, s_{2}, \ldots, s_{n}\right)^{T}$ be the weighted vector with condition $s_{j} \in[0,1], \sum_{j=1}^{n} s_{j}=1$, and $(\tau(1), \tau(2), \tau(3), \ldots, \tau(n))$ is any rearrangement of $(1,2,3, \ldots, n)$ such that $\rho_{\tau(j-1)} \geq \rho_{\tau(j)}$. 
Example 4: To develop the above method, we construct an example. For this we consider, $\rho_{1}=(0.60,0.30), \rho_{2}=(0.50,0.50), \rho_{3}=(0.30,0.50)$, and $\rho_{4}=(0.50,0.40)$ are four IFNs, $s=(0.40,0.30,0.20,0.10)$ be their weight vector, and $1_{1}=1_{2}=1_{3}=1_{4}=0.2$. First here we calculate the score function such as: $S\left(\rho_{1}\right)=(0.60,0.30)=0.30, S\left(\rho_{2}\right)=(0.50,0.50)=0.00$ $S\left(\rho_{3}\right)=(0.30,0.50)=-0.20, \quad S\left(\rho_{4}\right)=(0.50,0.40)=0.20 . \quad$ Hence,$\quad \rho_{\tau(1)}=(0.60,0.30)$ $\rho_{\tau(2)}=(0.50,0.40), \quad \rho_{\tau(3)}=(0.50,0.50)$ and $\rho_{\tau(4)}=(0.30,0.50)$. By applying LIFEWOG operator, we get

$$
\begin{aligned}
& \operatorname{LIFEOWG}_{s}\left(\rho_{1}, \rho_{2}, \rho_{3}, \rho_{4}\right) \\
& =\left(\begin{array}{l}
\frac{2\left(1-\log _{0.2}(0.60)\right)^{0.10}\left(1-\log _{0.2}(0.50)\right)^{0.30}\left(1-\log _{0.2}(0.50)\right)^{0.20}\left(1-\log _{0.2}(0.30)\right)^{0.40}}{\left(1+\log _{0.2}(0.60)\right)^{0.10}\left(1+\log _{0.2}(0.50)\right)^{0.30}\left(1+\log _{0.2}(0.50)\right)^{0.20}\left(1+\log _{0.2}(0.30)\right)^{0.40}}, \\
+\left(1-\log _{0.2}(0.60)\right)^{0.10}\left(1-\log _{0.2}(0.50)\right)^{0.30}\left(1-\log _{0.2}(0.50)\right)^{0.20}\left(1-\log _{0.2}(0.30)\right)^{0.40} \\
\left(1+\log _{0.2}(1-0.30)\right)^{0.10}\left(1+\log _{0.2}(1-0.40)\right)^{0.30}\left(1+\log _{0.2}(1-0.50)\right)^{0.20}\left(1+\log _{0.2}(1-0.50)\right)^{0.40} \\
\frac{\left(1-\log _{0.2}(1-0.30)\right)^{0.10}\left(1-\log _{0.2}(1-0.40)\right)^{0.30}\left(1-\log _{0.2}(1-0.50)\right)^{0.20}\left(1-\log _{0.2}(1-0.50)\right)^{0.40}}{\left(1+\log _{0.2}(1-0.30)\right)^{0.10}\left(1+\log _{0.2}(1-0.40)\right)^{0.30}\left(1+\log _{0.2}(1-0.50)\right)^{0.20}\left(1+\log _{0.2}(1-0.50)\right)^{0.40}} \\
+\left(1-\log _{0.2}(1-0.30)\right)^{0.10}\left(1-\log _{0.2}(1-0.40)\right)^{0.30}\left(1-\log _{0.2}(1-0.50)\right)^{0.20}\left(1-\log _{0.2}(1-0.50)\right)^{0.40}
\end{array}\right) \\
& =(0.42,0.39)
\end{aligned}
$$

Theorem 13: Let $\rho_{j}=\left(r_{j}, h_{j}\right)$ be a group of $n$ IFVs , then their resulting value by using LIFEOWG operator is also IFV.

Proof: The proof is parallel to Theorem 10.

Theorem 14: Let $\rho_{j}=\left(r_{j}, h_{j}\right)$ be a group of $n$ IFVs, then

$$
\operatorname{LIFOWG}_{s}\left(\rho_{1}, \rho_{2}, \ldots, \rho_{n}\right) \leq \operatorname{LIFEOWG}_{s}\left(\rho_{1}, \rho_{2}, \ldots, \rho_{n}\right)
$$

Proof: Proof is similar to Theorem 11.

Definition 15: Let $\rho_{j}=\left(r_{j}, h_{j}\right)$, where $\&_{j}=\rho_{j}{ }^{n \gamma_{j}}, \gamma=\left(\gamma_{1}, \gamma_{2}, \ldots, \gamma_{n}\right)^{T}$ be the weighted vector with condition $\gamma_{j}$ f $0, \sum_{j=1}^{n} \gamma_{j}=1$ and $n$ is a constant number. $s=\left(s_{1}, s_{2}, \ldots, s_{n}\right)^{T}$ be the associated vector with conditions $s_{j}$ f 0 and $\sum_{j=1}^{n} s_{j}=1$. Then the LIFEHG can be defined as: 


$$
\begin{aligned}
& \operatorname{LIFEHG}_{\gamma, s}\left(\rho_{1}, \rho_{2}, \ldots, \rho_{n}\right)
\end{aligned}
$$

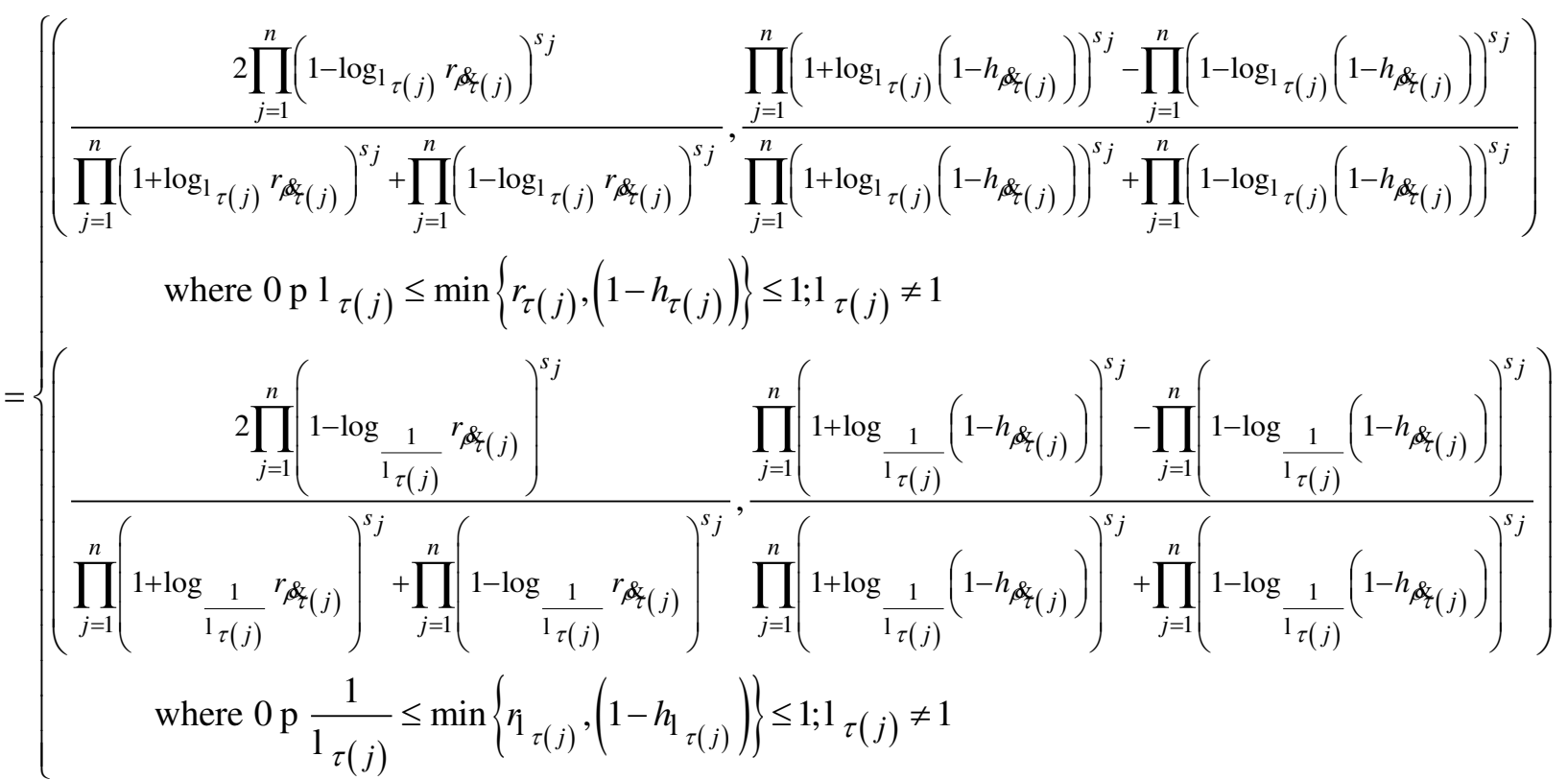

As similar to LIFEWG operator, the LIFEOWG operator and LIFEHG operator also have the same properties such as commutatively, idempotency, boundedness and monotonicity.

\section{An Application of the New Proposed Operators}

In this part, we consider a decision-making process under IFN information based on the novel proposed methods. Suppose a decision-making process which contains some different alternatives presented by $A=\left\{A_{1}, A_{2} \ldots, A_{m}\right\}$, which are calculated under different types criteria such as $C=\left\{C_{1}, C_{2}, \ldots, C_{n}\right\}$. The weighted vector of the criteria is presented by $s=\left(s_{1}, s_{2}, \ldots, s_{n}\right)^{T}$ with condition such as their sum is equal to 1 . Suppose there are $k$ experts for decision such as $E=\left\{E_{1}, E_{2}, \ldots, E_{k}\right\}$ and their weights are $\wp=\left(\wp_{1}, \wp_{2}, \ldots, \wp_{k}\right)^{T}$ with condition $\wp_{j} \in[0,1]$ and $\sum_{j=1}^{k} \wp_{j}=1$.

The major steps are the following:

Step 1: Construct decision matrices based on the expert's opinions.

Step 2: If the criteria have two types such as cost type's criteria and benefit type's criteria, then we, convert type's criteria into benefit type's criteria. Convert decision matrices into normalized decision matrices.

Step 3: Utilize the proposed operators to aggregate all individual matrices, into a single matrix.

Step 4: Again utilize all of the proposed methods to aggregate all the preference values.

Step 5: By Definition 3, we calculate the scores functions all the preference values. 
Step 6: Select that alternative, which has the highest score function.

\section{Illustrative Example}

In Pakistan, the ratio of Lower Respiratory Tract Infection (in short LRTI) was increasing day-by-day. But the Govt of Pakistan desired to regulates the ratio of this disease. For this Pakistan construct a committee of four experts whose weight vector is $\wp=(0.10,0.20,0.30 .40)^{T}$. The experts consider four alternatives $A_{1}$ : Augmentin, $A_{2}$ : Clathromycin, $A_{3}$ : Levofloxacin, $A_{4}$ : Moxiflaxacin. The experts take decision according to the following four criteria $\mathrm{C}_{1}$ : Disadvantages of the Medicine, $\mathrm{C}_{2}$ : Availability of the Medicine, $\mathrm{C}_{3}$ : Expenditure of the Medicine, $\mathrm{C}_{4}$ : Qualities of the Medicine, whose weighting vector is $s=(0.10,0.20,0.30,0.40)^{T}$. Here $C_{1}, C_{3}$ are cost type's criteria and $C_{2}, C_{4}$ are benefit type's criteria.

Step 1: In this step, we construct decision matrices for decision:

Table 1: Decision Matrix of Expert 1:

\begin{tabular}{|l|l|l|l|l|}
\hline & \multicolumn{2}{|l|}{$C_{1}$} & \multicolumn{1}{|l|}{$C_{2}$} & \multicolumn{1}{l|}{$C_{4}$} \\
\hline$A_{1}$ & $(0.40,0.50)$ & $(0.40,0.60)$ & $(0.50,0.40)$ & $(0.30,0.60)$ \\
\hline$A_{2}$ & $(0.30,0.60)$ & $(0.40,0.50)$ & $(0.40,0.60)$ & $(0.50,0.30)$ \\
\hline$A_{3}$ & $(0.50,0.40)$ & $(0.30,0.60)$ & $(0.40,0.50)$ & $(0.40,0.60)$ \\
\hline$A_{4}$ & $(0.40,0.60)$ & $(0.50,0.30)$ & $(0.30,0.60)$ & $(0.40,0.50)$ \\
\hline
\end{tabular}

Table 2: Decision Matrix of Expert 2:

\begin{tabular}{|l|l|l|l|l|}
\hline & \multicolumn{1}{|l|}{$C_{1}$} & \multicolumn{1}{|l|}{$C_{2}$} & \multicolumn{1}{l|}{$C_{4}$} \\
\hline$A_{1}$ & $(0.40,0.40)$ & $(0.30,0.40)$ & $(0.30,0.60)$ & $(0.50,0.50)$ \\
\hline$A_{2}$ & $(0.50,0.50)$ & $(0.40,0.40)$ & $(0.30,0.40)$ & $(0.40,0.40)$ \\
\hline$A_{3}$ & $(0.30,0.60)$ & $(0.50,0.50)$ & $(0.40,0.40)$ & $(0.30,0.40)$ \\
\hline$A_{4}$ & $(0.30,0.40)$ & $(0.40,0.40)$ & $(0.50,0.50)$ & $(0.40,0.40)$ \\
\hline
\end{tabular}

Table 3: Decision Matrix of Expert 3:

\begin{tabular}{|l|l|l|l|l|}
\hline & \multicolumn{1}{|l|}{$C_{1}$} & \multicolumn{1}{|l|}{$C_{2}$} & \multicolumn{1}{l|}{$C_{4}$} \\
\hline$A_{1}$ & $(0.30,0.70)$ & $(0.30,0.50)$ & $(0.40,0.60)$ & $(0.50,0.40)$ \\
\hline$A_{2}$ & $(0.40,0.60)$ & $(0.30,0.70)$ & $(0.30,0.50)$ & $(0.40,0.60)$ \\
\hline$A_{3}$ & $(0.40,0.60)$ & $(0.50,0.40)$ & $(0.30,0.70)$ & $(0.30,0.50)$ \\
\hline$A_{4}$ & $(0.30,0.50)$ & $(0.40,0.60)$ & $(0.40,0.60)$ & $(0.30,0.70)$ \\
\hline
\end{tabular}


Table 4: Decision Matrix of Expert 4:

\begin{tabular}{|l|l|l|l|l|}
\hline & \multicolumn{2}{|l|}{$C_{1}$} & \multicolumn{1}{|l|}{$C_{2}$} & \multicolumn{1}{l|}{$C_{4}$} \\
\hline$A_{1}$ & $(0.30,0.50)$ & $(0.40,0.50)$ & $(0.40,0.60)$ & $(0.40,0.40)$ \\
\hline$A_{2}$ & $(0.40,0.50)$ & $(0.30,0.70)$ & $(0.30,0.50)$ & $(0.40,0.60)$ \\
\hline$A_{3}$ & $(0.40,0.60)$ & $(0.40,0.40)$ & $(0.30,0.50)$ & $(0.40,0.50)$ \\
\hline$A_{4}$ & $(0.30,0.50)$ & $(0.40,0.60)$ & $(0.40,0.50)$ & $(0.30,0.70)$ \\
\hline
\end{tabular}

Step 2: In this step, we convert the cost type's criteria into benefit type's criteria.

Table 5: Normalized Decision Matrix of Expert 1:

\begin{tabular}{|c|c|c|c|c|}
\hline & $C_{1}$ & $C_{2}$ & $C_{3}$ & $C_{4}$ \\
\hline$A_{1}$ & $(0.50,0.40)$ & $(0.40,0.60)$ & $(0.40,0.50)$ & $(0.30,0.60)$ \\
\hline$A_{2}$ & $(0.60,0.30)$ & $(0.40,0.50)$ & $(0.60,0.40)$ & $(0.50,0.30)$ \\
\hline$A_{3}$ & $(0.40,0.50)$ & $(0.30,0.60)$ & $(0.50,0.40)$ & $(0.40,0.60)$ \\
\hline$A_{4}$ & $(0.60,0.40)$ & $(0.50,0.30)$ & $(0.60,0.30)$ & $(0.40,0.50)$ \\
\hline
\end{tabular}

Table 6: Normalized Decision Matrix of Expert 2:

\begin{tabular}{|c|c|c|c|c|} 
& $C_{1}$ & $C_{2}$ & $C_{3}$ & $C_{4}$ \\
\hline$A_{1}$ & $(0.40,0.40)$ & $(0.30,0.40)$ & $(0.60,0.30)$ & $(0.50,0.50)$ \\
\hline$A_{2}$ & $(0.50,0.50)$ & $(0.40,0.40)$ & $(0.40,0.30)$ & $(0.40,0.40)$ \\
\hline$A_{3}$ & $(0.60,0.30)$ & $(0.50,0.50)$ & $(0.40,0.40)$ & $(0.30,0.40)$ \\
\hline$A_{4}$ & $(0.40,0.30)$ & $(0.40,0.40)$ & $(0.50,0.50)$ & $(0.40,0.40)$ \\
\hline
\end{tabular}

Table 7: Normalized Decision Matrix of Expert 3:

\begin{tabular}{|c|c|c|c|c|}
\hline & $C_{1}$ & $C_{2}$ & $C_{3}$ & $C_{4}$ \\
\hline$A_{1}$ & $(0.70,0.30)$ & $(0.30,0.50)$ & $(0.60,0.40)$ & $(0.50,0.40)$ \\
\hline$A_{2}$ & $(0.60,0.40)$ & $(0.30,0.70)$ & $(0.50,0.30)$ & $(0.40,0.60)$ \\
\hline$A_{3}$ & $(0.60,0.40)$ & $(0.50,0.40)$ & $(0.70,0.30)$ & $(0.30,0.50)$ \\
\hline$A_{4}$ & $(0.50,0.30)$ & $(0.40,0.60)$ & $(0.60,0.40)$ & $(0.30,0.70)$ \\
\hline
\end{tabular}

Table 8: Normalized Decision Matrix of Expert 4:

\begin{tabular}{|c|c|c|c|c|}
\hline & $C_{1}$ & $C_{2}$ & $C_{3}$ & $C_{4}$ \\
\hline$A_{1}$ & $(0.50,0.30)$ & $(0.40,0.50)$ & $(0.60,0.40)$ & $(0.40,0.40)$ \\
\hline$A_{2}$ & $(0.50,0.40)$ & $(0.30,0.70)$ & $(0.50,0.30)$ & $(0.40,0.60)$ \\
\hline$A_{3}$ & $(0.60,0.40)$ & $(0.40,0.40)$ & $(0.50,0.30)$ & $(0.40,0.50)$ \\
\hline$A_{4}$ & $(0.50,0.30)$ & $(0.40,0.60)$ & $(0.50,0.40)$ & $(0.30,0.70)$ \\
\hline
\end{tabular}


Step 3: Utilize the LIFEWA operator, where $\wp=(0.10,0.20,0.30 .40)^{T}$ and $1=0.2$, we have

Table 9: Collective Normalized Averaging Matrix $R$

\begin{tabular}{|c|c|c|c|c|}
\hline & $\mathrm{C}_{1}$ & $\mathrm{C}_{2}$ & $\mathrm{C}_{3}$ & $\mathrm{C}_{4}$ \\
\hline$A_{1}$ & $(0.630,0.256)$ & $(0.547,0.316)$ & $(0.663,0.304)$ & $(0.521,0.357)$ \\
\hline$A_{2}$ & $(0.624,0.314)$ & $(0.482,0.296)$ & $(0.558,0.229)$ & $(0.546,0.410)$ \\
\hline$A_{3}$ & $(0.665,0.321)$ & $(0.591,0.357)$ & $(0.628,0.246)$ & $(0.547,0.316)$ \\
\hline$A_{4}$ & $(0.568,0.234)$ & $(0.536,0.460)$ & $(0.619,0.324)$ & $(0.596,0.382)$ \\
\hline
\end{tabular}

Again utilize the LIFEWG operator, where $\wp=(0.10,0.20,0.30 .40)^{T}$ and $1=0.2$, we have

Table 10: Collective Normalized Geometric Matrix $R$

\begin{tabular}{ccccc}
\hline & $\mathrm{C}_{1}$ & $\mathrm{C}_{2}$ & $\mathrm{C}_{3}$ & $\mathrm{C}_{4}$ \\
\hline$A_{1}$ & $(0.624,0.216)$ & $(0.544,0.312)$ & $(0.667,0.324)$ & $(0.527,0.351)$ \\
$A_{2}$ & $(0.614,0.310)$ & $(0.478,0.291)$ & $(0.558,0.229)$ & $(0.546,0.410)$ \\
$A_{3}$ & $(0.665,0.321)$ & $(0.593,0.357)$ & $(0.628,0.246)$ & $(0.547,0.316)$ \\
$A_{4}$ & $(0.563,0.224)$ & $(0.532,0.458)$ & $(0.623,0.322)$ & $(0.601,0.362)$ \\
\hline
\end{tabular}

Step 4: From Table 9 and Table 10, we computing the hybrid values such as, $\&_{j}=n \gamma_{j}\left(\rho_{j}\right)$, $\&_{j}=\left(\rho_{j}\right)^{n \gamma_{j}}$, where $\gamma=(0.10,0.20,0.30,0.40)^{T}$ and get Tables 11 and Tables 12 respectively:

Table 11: Hybrid Averaging Matrix $R$

\begin{tabular}{lcccc}
\hline & $\mathrm{C}_{1}$ & $\mathrm{C}_{2}$ & $\mathrm{C}_{3}$ & $\mathrm{C}_{4}$ \\
\hline$A_{1}$ & $(0.570,0.226)$ & $(0.588,0.295)$ & $(0.728,0.239)$ & $(0.660,0.192)$ \\
$A_{2}$ & $(0.627,0.320)$ & $(0.660,0.319)$ & $(0.624,0.170)$ & $(0.611,0.288)$ \\
$A_{3}$ & $(0.621,0.352)$ & $(0.477,0.238)$ & $(0.694,0.185)$ & $(0.494,0.245)$ \\
$A_{4}$ & $(0.578,0.254)$ & $(0.676,0.337)$ & $(0.685,0.258)$ & $(0.536,0.336)$ \\
\hline
\end{tabular}

Table 12: Hybrid Geometric Matrix $R$

\begin{tabular}{lcccc}
\hline & $\mathrm{C}_{1}$ & $\mathrm{C}_{2}$ & $\mathrm{C}_{3}$ & $\mathrm{C}_{4}$ \\
\hline$A_{1}$ & $(0.714,0.258)$ & $(0.524,0.389)$ & $(0.562,0.374)$ & $(0.765,0.214)$ \\
$A_{2}$ & $(0.830,0.106)$ & $(0.528,0.348)$ & $(0.610,0.352)$ & $(0.520,0.306)$ \\
$A_{3}$ & $(0.825,0.144)$ & $(0.479,0.315)$ & $(0.464,0.268)$ & $(0.574,0.226)$ \\
$A_{4}$ & $(0.848,0.134)$ & $(0.566,0.297)$ & $(0.572,0.287)$ & $(0.577,0.202)$ \\
\hline
\end{tabular}

Step 5: Using (Table 9, 10, 11, 12): and utilize the different operators to aggregate all preference values, where $\gamma=(0.10,0.20,0.30,0.40)^{T}, s=(0.10,0.20,0.30,0.40)^{T}$, and get the following: 
Table13: All Preference values of different operators

\begin{tabular}{|l|l|l|l|l|l|l|}
\hline & LIFEWA & LIFEOWA & LIFEHA & LIFEWG & LIFEOWG & LIFEHG \\
\hline$A_{1}$ & $(0.537,0.328)$ & $(0.494,0.286)$ & $(0.449,0.220)$ & $(0.604,0.257)$ & $(0.546,0.273)$ & $(0.489,0.243)$ \\
\hline$A_{2}$ & $(0.588,0.325)$ & $(0.491,0.229)$ & $(0.489,0.297)$ & $(0.549,0.311)$ & $(0.525,0.381)$ & $(0.497,0.258)$ \\
\hline$A_{3}$ & $(0.523,0.295)$ & $(0.498,0.287)$ & $(0.496,0.221)$ & $(0.559,0.255)$ & $(0.546,0.273)$ & $(0.581,0.345)$ \\
\hline$A_{4}$ & $(0.494,0.214)$ & $(0.488,0.239)$ & $(0.510,0.218)$ & $(0.598,0.224)$ & $(0.525,0.381)$ & $(0.522,0.221)$ \\
\hline
\end{tabular}

Table14: Score functions of all different operators

\begin{tabular}{|c|c|c|}
\hline Operators & Score functions & Ranking \\
\hline LIFEWA & $0.209,0.263,0.228,0.280$ & $A_{4} \mathrm{f} A_{2} \mathrm{f} A_{3} \mathrm{f} A_{1}$ \\
\hline LIFEOWA & $0.208,0.262,0.211,0.269$ & $A_{4} \mathrm{f} A_{2} \mathrm{f} A_{3} \mathrm{f} A_{1}$ \\
\hline LIFEHA & $0.192,0.285,0.279,0.292$ & $A_{4} \mathrm{f} A_{2} \mathrm{f} A_{3} \mathrm{f} A_{1}$ \\
\hline LIFEWG & $0.347,0.238,0.304,0.374$ & $A_{4} \mathrm{f} A_{1} \mathrm{f} A_{3} \mathrm{f} A_{2}$ \\
\hline LIFEOWG & $0.273,0.144,0.272,0.324$ & $A_{4} \mathrm{f} A_{1} \mathrm{f} A_{3} \mathrm{f} A_{2}$ \\
\hline LIFEHG & $0.246,0.231,0.240,0.301$ & $A_{4} \mathrm{f} A_{1} \mathrm{f} A_{3} \mathrm{f} A_{2}$ \\
\hline
\end{tabular}

\section{Ranking of different operators}

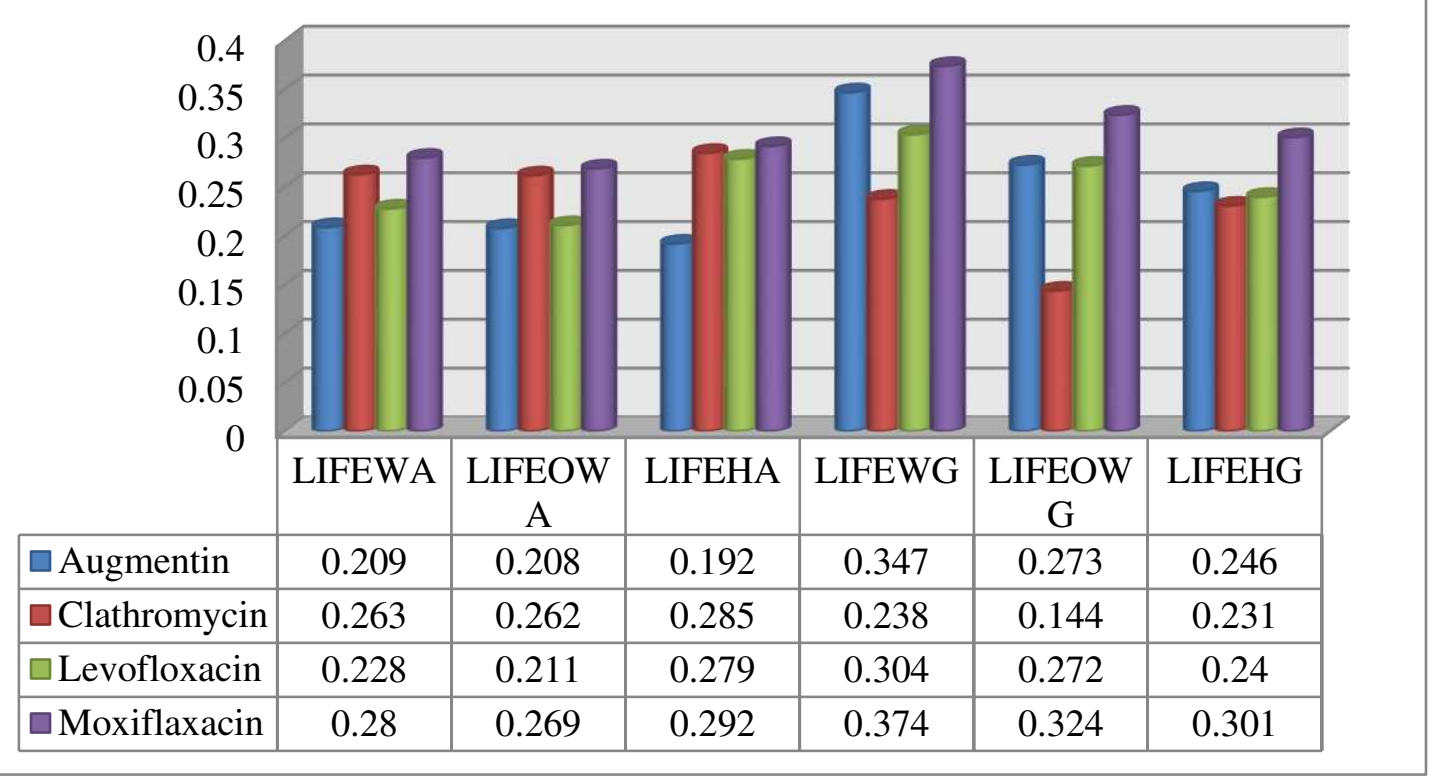

Fig 1: 
Table15: Comparisons with existing methods

\begin{tabular}{|c|c|c|c|}
\hline Averaging operators & Ordering & Geometric operators & Ordering \\
\hline IFEWA $^{\text {(Wang and Liu , 2012) }}$ & $A_{4} \mathrm{f} A_{2} \mathrm{f} A_{1} \mathrm{f} A_{3}$ & IFEWG $^{\text {(Wang and Liu , 2011) }}$ & $A_{4} \mathrm{f} A_{2} \mathrm{f} A_{1} \mathrm{f} A_{3}$ \\
\hline IFEOWA $^{\text {(Wang and Liu, 2012) }}$ & $A_{4} \mathrm{f} A_{2} \mathrm{f} A_{1} \mathrm{f} A_{3}$ & IFEOWG $^{\text {(Wang and Liu, 2011) }}$ & $A_{4} \mathrm{f} A_{2} \mathrm{f} A_{1} \mathrm{f} A_{3}$ \\
\hline IFEHA $^{\text {(Zhao and Wei , 2013) }}$ & $A_{4} \mathrm{f} A_{2} \mathrm{f} A_{1} \mathrm{f} A_{3}$ & IFEHG $^{\text {(Zhao and Wei , 2013) }}$ & $A_{4} \mathrm{f} A_{2} \mathrm{f} A_{1} \mathrm{f} A_{3}$ \\
\hline $\mathrm{LIFWA}^{(\mathrm{Li} \text { and Wei, 2017) }}$ & $A_{4} \mathrm{f} A_{1} \mathrm{f} A_{3} \mathrm{f} A_{2}$ & $\mathrm{LIFWG}^{(\mathrm{Li} \text { and Wei , 2017) }}$ & $A_{4} \mathrm{f} A_{1} \mathrm{f} A_{3} \mathrm{f} A_{2}$ \\
\hline LIFOWA $^{(\mathrm{Li} \text { and Wei, 2017) }}$ & $A_{4} \mathrm{f} A_{1} \mathrm{f} A_{3} \mathrm{f} A_{2}$ & $\mathrm{LIFOWG}^{(\mathrm{Li} \text { and Wei , 2017) }}$ & $A_{4} \mathrm{f} A_{1} \mathrm{f} A_{3} \mathrm{f} A_{2}$ \\
\hline $\mathrm{IFWA}^{(\mathrm{Xu}, 2007)}$ & $A_{4} \mathrm{f} A_{1} \mathrm{f} A_{2} \mathrm{f} A_{3}$ & $\mathrm{IFWG}^{(\mathrm{Xu} \text { and Yager, 2006) }}$ & $A_{4} \mathrm{f} A_{1} \mathrm{f} A_{2} \mathrm{f} A_{3}$ \\
\hline IFOWA $^{((X u, 2007)}$ & $A_{4} \mathrm{f} A_{1} \mathrm{f} A_{2} \mathrm{f} A_{3}$ & IFOWG $^{(\mathrm{Xu} \text { and Yager, 2006) }}$ & $A_{4} \mathrm{f} A_{1} \mathrm{f} A_{2} \mathrm{f} A_{3}$ \\
\hline IFHA $^{(X u, 2007)}$ & $A_{4} \mathrm{f} A_{1} \mathrm{f} A_{2} \mathrm{f} A_{3}$ & $\mathrm{IFHG}^{(\mathrm{Xu} \text { and Yager, 2006) }}$ & $A_{4} \mathrm{f} A_{1} \mathrm{f} A_{2} \mathrm{f} A_{3}$ \\
\hline LIFEWA & $A_{4} \mathrm{f} A_{2} \mathrm{f} A_{3} \mathrm{f} A_{1}$ & LIFEWG & $A_{4} \mathrm{f} A_{1} \mathrm{f} A_{3} \mathrm{f} A_{2}$ \\
\hline LIFEOWA & $A_{4} \mathrm{f} A_{2} \mathrm{f} A_{3} \mathrm{f} A_{1}$ & LIFEOWG & $A_{4} \mathrm{f} A_{1} \mathrm{f} A_{3} \mathrm{f} A_{2}$ \\
\hline LIFEHA & $A_{4} \mathrm{f} A_{2} \mathrm{f} A_{3} \mathrm{f} A_{1}$ & LIFEHG & $A_{4} \mathrm{f} A_{1} \mathrm{f} A_{3} \mathrm{f} A_{2}$ \\
\hline
\end{tabular}

\section{Limitations:}

Einstein sum and Einstein product are the good alternative of algebraic sum and product. Therefore in this paper, we introduced some novel Einstein operational laws under intuitionistic fuzzy environment, which can overcome the weaknesses of algebraic operational laws, and then developed several Einstein aggregation operators, which can overcome the algebraic operator's drawbacks.

However, these novel methods have some limitations such as, $\log _{1}(0)$ and $\log _{1}(y)$ are not defined in real numbers. Let $\rho$ be an IFN and 1 be a real number, then $\log _{1}(\rho)$, with $\rho=0$ and $\log _{1}(\rho)$ with $1=1$ cannot be defined. So, these are the limitations of the proposed methods. Therefore throughout in this paper, we assume that $\rho \neq 0$ and $1 \neq 1$.

\section{Conclusion}

In this paper, we have developed a new logarithmic operational law of intuitionistic fuzzy set and intuitionistic fuzzy numbers with real number 1 with $1 \in(0,1)$. Additionally, we have also investigated their properties and relationships. Base on the new logarithmic operational laws, we have investigated some averaging and geometric aggregation operators named as LIFEWA 
operator, LIFEOWA operator, LIFEHA operator, LIFEWG operator, LIFEOWG operator and LIFEHG operator along with their some basic properties such as idempotency, boundedness, monotonicity. Finally, the new proposed methods have been investigated to multicriteria decision-making problem with intuitionistic fuzzy information to prove their legitimacy, suitability and efficiency. Thus, we also demonstrated the superiority and the validity of the proposed aggregation operators using some of the existing methods. Hence, the analysis showed that our proposed aggregation operator is more reliable and accurate than the existing methods. Thus, our proposed logarithmic aggregation operator provides new way to find the best alternative in the MADM decision-making problem.

For further study, it is needed to give the advantages of the proposed operators to the other fields, such as inducing variables, pattern recognition, symmetric operators, power operators, Hamacher operator, Dombi operators, uncertain programming, Linguistic terms, confidence levels interval-valued etc.

\section{Compliance with ethical standards}

Conflict of interest: The authors declare that they have no conflict of interest.

Ethical approval: This article does not contain any studies with human participants or animals performed by any of the authors.

\section{References}

Atanassov, K. T. (1986). Intuitionistic fuzzy sets. Fuzzy Sets and Systems, 20(1), 87-96.

Atanassov, K.T. (1989). More on intuitionistic fuzzy sets. Fuzzy Sets and Systems, 33(1), $37-46$

Dalman, H., Guzel, N., and Sivri, M. (2016). A fuzzy set-based approach to multi objective multi-item solid transportation problem under uncertainty. Int J Fuzzy Syst, 18 (4), 716729

Ding Y, Huang L, Li S, Gao H, Deng H, Li Y, Liu G (2020) Definition and Application of Variable Resistance Coefficient for Wheeled Mobile Robots on Deformable Terrain. IEEE transactions on robotics 36: 894 - 909

Garg, H. (2016a). Generalized intuitionistic fuzzy interactive geometric interaction operators using Einstein t-norm and t-conorm and their application to decision making. Comput Ind Eng, 101, 53-69 
Garg, H. (2016b). Generalized intuitionistic fuzzy multiplicative interactive geometric operators and their application to multiple criteria decision making. Int J Mach Learn Cybern, 7 (6), 1075-1092

Garg, H. (2016c). A new generalized improved score function of interval-valued intuitionistic fuzzy sets and applications in expert systems. Appl Soft Comput, 38, 988-999

Garg, H., Agarwal, N., and Tripathi, A. (2015). Entropy based multi-criteria decision making method under fuzzy environment and unknown attribute weights. Glob J Technol Optim $6(3), 13-20$

Garg, H., and Nancy. (2016d). An improved score function for ranking neutrosophic sets and its application to decision-making process. Int J Uncertain Quantif, 6, 377-385

Gou, X.J., Xu, Z.S., and Lei, Q. (2016). New operational laws and aggregation method of intuitionistic fuzzy information. Journal of Intelligent \& Fuzzy Systems, 30 (1), 129141.

He Y, Dai L, Zhang H (2020) Multi-Branch Deep Residual Learning for Clustering and Beamforming in User-Centric Network. IEEE communications letters 24: 2221-2225

Kumar, K., and Garg, H. (2016). TOPSIS method based on the connection number of set pair analysis under interval-valued intuitionistic fuzzy set environment. Comput Appl Math, $37(2), 1319-1329$

Kordestani H, Zhang C, Masri S F, Shadabfar M (2021) An empirical time-domain trend linebased bridge signal decomposing algorithm using Savitzky-Golay filter. Structural control and health monitoring 28: 1-24.

Li, Z., and Wei, F. (2017). The logarithmic operational laws of intuitionistic fuzzy sets and intuitionistic fuzzy numbers. Journal of Intelligent \& Fuzzy Systems, 33 (6), 3241-3253

Li B, Feng Y, Xiong Z, Yang W, Liu G (2021) Research on AI security enhanced encryption algorithm of autonomous IoT systems. Information sciences 575: 379-398

Meng F, Wang D, Yang P, Xie G (2019) Application of Sum of Squares Method in Nonlinear $\mathrm{H}_{\infty}$ Control for Satellite Attitude Maneuvers. Complexity https://doi.org/10.1155/2019/5124108.

Nancy, and Garg, H. (2016e). Novel single-valued neutrosophic decision making operators under frank norm operations and its application. Int J Uncertain Quantif, 6, 361-375 
Rahman, K., Abdullah, S., Jamil, M., and Muhammad, Y.K. (2018). Some generalized intuitionistic fuzzy Einstein hybrid aggregation operators and their application to multiple-attribute group decision-making. International Journal of Fuzzy Systems, 20(5), 1567-1575.

Sun G, Cong g, Dong J, Liu Y, Ding Z, Yu H (2021) What and How: Generalized Lifelong Spectral Clustering via Dual Memory. IEEE transactions on pattern analysis and machine intelligence DOI: 10.1109/TPAMI.2021.3058852

Sun G, Cong Y, Wang Q, Zhong B, Fu Y (2015) Representative Task Self-selection for Flexible Clustered Lifelong Learning. IEEE transaction on neural networks and learning systems 14: $1-15$

Wang, W., and Liu, X. (2011). Intuitionistic Fuzzy Geometric Aggregation Operators Based on Einstein Operations. International journal of intelligent systems, 26(11), 1049-1075.

Wang, W., and Liu, X. (2012). Intuitionistic fuzzy information aggregation using Einstein operations. IEEE Trans. Fuzzy Syst, 20(5), 923-938.

Xu, Z.S. (2007). Intuitionistic fuzzy aggregation operators. IEEE Trans Fuzzy Syst, 15(6), 11791187

Xu, Y., H. Wang, H., and Merigo, J.M. (2014). Intuitionistic fuzzy Einstein choquet integral operators for multiple attribute decision making. Technol Econ Dev Econ 20 (2), 227253

Xu, Z.S., and Yager, R.R. (2006). Some geometric aggregation operators based on intuitionistic fuzzy sets, Int J Gen Syst 35(4), 417-433

Yager, R.R. (1988). On ordered weighted averaging aggregation operators in multi criteria decision making. IEEE Trans Syst Man Cybern, 18(1), 183-190

Yager, R.R., and Kacprzyk, J. (1997). The ordered weighted averaging operators: theory and applications, Kluwer Academic Publisher, Boston

Ye, J. (2007). Improved method of multi criteria fuzzy decision making based on vague sets. Comput Aided Des, 39(4), 164-169

Ye, J. (2009). Multicriteria fuzzy decision-making method based on a novel accuracy function under interval-valued intuitionistic fuzzy environment. Expert Syst Appl, 36(6), 09-6902

$\mathrm{Yu}$, D. (2014). Intuitionistic fuzzy information aggregation under confidence levels, Appl Soft Comput, 19, 147-160 
Yu, D. (2015). A scientometrics review on aggregation operator research. Scientometrics 105 (1), 115-133

Yu, D., and Shi, S. (2015). Researching the development of Atanassov intuitionistic fuzzy set: using a citation network analysis. Appl Soft Comput 32(c), 189-198

Yang W, Chen X, Xiong Z, Xu Z, Liu G, Zhang X (2021) A privacy-preserving aggregation scheme based on negative survey for vehicle fuel consumption data. Information sciences 570: 526-544

Zadeh, L.A. (1965). Fuzzy sets. Inf. Control, 8(3), 338-353.

Zhao, X., and Wei, G. (2013). Some intuitionistic fuzzy Einstein hybrid aggregation operators and their application to multiple attribute decision making. Knowledge Based System, 37 , 472-479

Zhang Y, Liu F, Fang, Z, Yuan B, Zhang G, Lu J (2021) Learning From a ComplementaryLabel Source Domain: Theory and Algorithms. IEEE transaction on neural networks and learning systems DOI: 10.1109/TNNLS.2021.3086093

Zhao C, Zhong S, Zhong Q, Shi K (2020) Synchronization of Markovian complex networks with input mode delay and Markovian directed communication via distributed dynamic eventtriggered control. Nonlinear analysis Hybrid systems DOI: 10.1016/j.nahs.2020.100883 